\title{
NONRELATIVISTIC LIMIT OF SOLITARY WAVES FOR NONLINEAR MAXWELL-KLEIN-GORDON EQUATIONS
}

\author{
SANGDON JIN AND JINMYOUNG SEOK
}

\begin{abstract}
We study the nonrelativistic limit of solitary waves from Nonlinear Maxwell-KleinGordon equations (NMKG) to Nonlinear Schrödinger-Poisson equations (NSP). It is known that the existence or multiplicity of positive solutions depends on the choices of parameters the equations contain. In this paper, we prove that for a given positive solitary wave of NSP, which is found in Ruiz's work [19, there corresponds a family of positive solitary waves of NMKG under the nonrelativistic limit. Notably, our results contain a new result of existence of positive solutions to (NMKG) with lower order nonlinearity.
\end{abstract}

\section{INTRODUCTION}

Nonlinear Maxwell-Klein-Gordon equations are written by

$$
\left\{\begin{array}{l}
D_{\alpha} D^{\alpha} \phi=(m c)^{2} \phi-|\phi|^{p-2} \phi, \\
\partial^{\beta} F_{\alpha \beta}=\frac{q}{c} \operatorname{Im}\left(\phi \overline{D_{\alpha} \phi}\right),
\end{array} \quad \text { in } \mathbb{R}^{1+3} .\right.
$$

where $D_{\alpha}:=\partial_{\alpha}+\frac{q}{c} i A_{\alpha}, \alpha=0,1,2,3$ and $F_{\alpha \beta}:=\partial_{\alpha} A_{\beta}-\partial_{\beta} A_{\alpha}$. Here, $m>0$ represents the mass of a particle, $q>0$ is a unit charge and $c>0$ is the speed of light. We write $\partial_{0}=\frac{\partial}{c \partial t}$, $\partial_{i}=\frac{\partial}{\partial x_{j}}, j=1,2,3$. Indices are raised under the Minkowski metric $g_{\alpha \beta}=\operatorname{diag}(-1,1,1,1)$, i.e., $X^{\alpha}:=g_{\alpha \beta} X_{\beta}$. If we pay attention to the electrostatic situation, that is, $A_{1}=A_{2}=A_{3}=0$, then NMKG is reduced to

$$
\left\{\begin{array}{l}
\left(-\frac{\partial^{2}}{c^{2} \partial t^{2}}+\Delta\right) \phi-\frac{2 q}{c^{2}} i A_{0} \frac{\partial \phi}{\partial t}-\frac{q}{c^{2}} i \frac{\partial A_{0}}{\partial t} \phi+\left(\frac{q}{c}\right)^{2} A_{0}^{2} \phi=(m c)^{2} \phi-|\phi|^{p-2} \phi, \\
-\Delta A_{0}=\frac{q}{c^{2}} \operatorname{Im}\left(\phi \frac{\partial \phi}{\partial t}\right)-\left(\frac{q}{c}\right)^{2} A_{0}|\phi|^{2}
\end{array} \text { in } \mathbb{R}^{1+3} .\right.
$$

This paper is concerned with the nonrelativistic limit for NMKG in electrostatic case. By modulating the solution as $\phi(t, x)=e^{i m c^{2} t} \psi(t, x)$, the system of equations (11) transforms into

$$
\left\{\begin{array}{l}
-\frac{\partial^{2} \psi}{c^{2} \partial t^{2}}-2 m i \frac{\partial \psi}{\partial t}+\Delta \psi+2 q m A_{0} \psi-\frac{2 q}{c^{2}} i A_{0} \frac{\partial \psi}{\partial t}-\frac{q}{c^{2}} i \frac{\partial A_{0}}{\partial t} \psi+\left(\frac{q}{c}\right)^{2} A_{0}^{2} \psi=-|\psi|^{p-2} \psi, \\
-\Delta A_{0}+\left(\frac{q}{c}\right)^{2}|\psi|^{2} A_{0}=\frac{q}{c^{2}} \operatorname{Im}\left(\psi \frac{\partial \psi}{\partial t}\right)-q m|\psi|^{2} .
\end{array}\right.
$$

Then, taking so-called nonrelativistic limit $c \rightarrow \infty$, the relativistic system (2) formally converges to nonlinear equations of Schrödinger type, called the nonlinear Schrödinger-Poisson equations

$$
\left\{\begin{array}{l}
-2 m i \frac{\partial \psi}{\partial t}+\Delta \psi+2 q m A_{0} \psi=-|\psi|^{p-2} \psi, \quad \text { in } \mathbb{R}^{1+3} . \\
-\Delta A_{0}=-q m|\psi|^{2}
\end{array}\right.
$$

When the nonlinear potential term $|\psi|^{p-2} \psi$ is absent, the rigorous justifications of this limit are carried out by Masmoudi-Nakanish [16] and Bechouche-Mauser-Selberg [4. As for the stuides on the nonlinear Klein-Gordon equations without the Maxwell gauge terms $\left(A_{\mu}=0, \mu=0,1,2,3\right)$, we refer to a series for works [14, 15, 17.

The main interest of this paper lies in investigating the correspondence between solitary waves of NMKG and NSP under the nonrelativistic limit $c \rightarrow \infty$. During recent two decades, existence

Key words and phrases. Maxwell-Klein-Gordon; Schrodinger-Poisson; nonrelativistic limit; solitary wave. 
theories for solitary waves of NMKG and NSP have been well developed. Inserting the standing wave ansatz $\psi(t, x)=e^{-i \mu t} u(x), u \in \mathbb{R}$ into (2), we get

$$
\left\{\begin{array}{l}
-\Delta u+\left(m^{2} c^{2}-\left(\frac{m c^{2}-\mu}{c}+\frac{q \Phi}{c}\right)^{2}\right) u-|u|^{p-2} u=0, \\
-\Delta \Phi+\frac{q^{2}}{c^{2}} u^{2} \Phi=-\frac{q}{c}\left(\frac{m c^{2}-\mu}{c}\right) u^{2},
\end{array} \text { in } \mathbb{R}^{3} .\right.
$$

Lax-Milgram theorem implies that for each $u \in H^{1}\left(\mathbb{R}^{3}\right)$, there exists a unique solution $\Phi_{u} \in D^{1,2}\left(\mathbb{R}^{3}\right)$ of

$$
-\Delta \Phi+\frac{q^{2}}{c^{2}} u^{2} \Phi=-q\left(m-\frac{\mu}{c^{2}}\right) u^{2} \text { in } \mathbb{R}^{3} .
$$

Then, by [6. Proposition 3.5], $(u, \Phi) \in H^{1}\left(\mathbb{R}^{3}\right) \times D^{1,2}\left(\mathbb{R}^{3}\right)$ is a solution of (3) if and only if $u \in H^{1}\left(\mathbb{R}^{3}\right)$ is a critical point of $I_{c}$, and $\Phi=\Phi_{u}$, where

$$
I_{c}(u)=\frac{1}{2} \int_{\mathbb{R}^{3}}|\nabla u|^{2}+\left(2 m \mu-\frac{\mu^{2}}{c^{2}}\right) u^{2}-q\left(m-\frac{\mu}{c^{2}}\right) u^{2} \Phi_{u} d x-\frac{1}{p} \int_{\mathbb{R}^{3}}|u|^{p} d x,
$$

which is a $C^{1}$ functional on $H^{1}\left(\mathbb{R}^{3}\right)$. We note that the system of equations (3) is equivalent to the single nonlocal equation

$$
-\Delta u+\left(m^{2} c^{2}-\left(\frac{m c^{2}-\mu}{c}+\frac{q \Phi_{u}}{c}\right)^{2}\right) u-|u|^{p-2} u=0 \text { in } \mathbb{R}^{3} .
$$

Before stating the existence results for (3), we simplify the parameters by denoting $\bar{m}=m c$, $e=q / c$ and $\omega=\left(m c^{2}-\mu\right) / c$ to rewrite (3) as

$$
\left\{\begin{array}{l}
-\Delta u+\left(\bar{m}^{2}-(\omega+e \Phi)^{2}\right) u-|u|^{p-2} u=0, \\
-\Delta \Phi+e^{2} u^{2} \Phi=-e \omega u^{2},
\end{array} \text { in } \mathbb{R}^{3},\right.
$$

where $e>0, \bar{m}>0$ and $\omega>0$ such that $\bar{m}>\omega$. The corresponding action functional is given by

$$
I_{\bar{m}, e, \omega}(u)=\frac{1}{2} \int_{\mathbb{R}^{3}}|\nabla u|^{2}+\left(\bar{m}^{2}-\omega^{2}\right) u^{2}-e \omega u^{2} \Phi_{u} d x-\frac{1}{p} \int_{\mathbb{R}^{3}}|u|^{p} d x .
$$

For fixed $e>0$, Benci and Fortunato [6] first proved by applying critical point theory to $I_{\bar{m}, e, \omega}$ that there exist infinitely many solutions of (6) for $4<p<6$ and $0<\omega<\bar{m}$. This result is extended by D'Aprile and Mugnai [1] to the cases $4 \leq p<6$ and $0<\omega<\bar{m}$ or $2<p<4$ and $0<\sqrt{2} \omega<\bar{m} \sqrt{p-2}$. They also proved in [12] that there exist no nontrivial solutions if $p \leq 2$ or $p \geq 6$ and $0<\omega \leq \bar{m}$. In [3], Azzollini, Pisani and Pomponio widened the existence range of $\bar{m}, \omega$ for the case $2<p<4$ by showing that (6) admits a nontrivial solution when $0<\omega<\bar{m} g(p)$, where

$$
g(p):=\left\{\begin{aligned}
\sqrt{(p-2)(4-p)} & \text { if } 2<p<3 \\
1 & \text { if } 3 \leq p<4 .
\end{aligned}\right.
$$

Azzollini and Pomponio also focused on the existence of a ground state solution of (6). A critical point of $I_{\bar{m}, e, \omega}$ is said to be a ground state solution to (6) if it minimizes the value of $I_{\bar{m}, e, \omega}$ among all nontrivial critical points of $I_{\bar{m}, e, \omega}$. In [2], they showed (6) admits a ground state solution if $4 \leq p<6$ and $0<\omega<\bar{m}$ or $2<p<4$ and $\bar{m} \sqrt{p-1}>\omega \sqrt{5-p}$. Wang [21] established the same result to the range of parameters that $2<p<4$ and $0<\sqrt{h(p)} \omega<\bar{m}$, where

$$
h(p):=1+\frac{(4-p)^{2}}{4(p-2)} .
$$

We now turn to the standing wave solutions for NSP. We again insert the same ansatz $\psi(t, x)=$ $e^{-i \mu t} u(x), u \in \mathbb{R}$ into NSP to obtain

$$
\begin{gathered}
-\Delta u+2 m \mu u-2 q m u \phi-|u|^{p-2} u=0 \text { in } \mathbb{R}^{3}, \\
-\Delta \phi=-q m u^{2} \text { in } \mathbb{R}^{3} .
\end{gathered}
$$

For any $u \in H^{1}\left(\mathbb{R}^{3}\right)$, there exists a unique $\phi_{u} \in D^{1,2}\left(\mathbb{R}^{3}\right)$ satisfying

$$
-\Delta \phi_{u}=-q m u^{2} \text { in } \mathbb{R}^{3} \text {, }
$$


by Lax-Milgram theorem (note that actually $\phi_{u}=-\frac{q m}{4 \pi|x|} * u^{2}$ ). We define the corresponding action integral as

$$
I_{\infty}(u)=\frac{1}{2} \int_{\mathbb{R}^{3}}|\nabla u|^{2}+2 m \mu u^{2}-q m u^{2} \phi_{u} d x-\frac{1}{p} \int_{\mathbb{R}^{3}}|u|^{p} d x .
$$

Then, by [11, Lemma 3.2], $(u, \phi) \in H^{1}\left(\mathbb{R}^{3}\right) \times D^{1,2}\left(\mathbb{R}^{3}\right)$ is a solution of (7) if and only if $u \in H^{1}\left(\mathbb{R}^{3}\right)$ is a critical point of $I_{\infty}$, and $\phi=\phi_{u}$. It is also standard to show that $I_{\infty} \in C^{1}\left(H^{1}\left(\mathbb{R}^{3}\right), \mathbb{R}\right)$ and a critical point $u$ of $I_{\infty}$ satisfies

$$
-\Delta u+2 m \mu u-2 q m u \phi_{u}-|u|^{p-2} u=0 \text { in } \mathbb{R}^{3} .
$$

We summarize some existence results for problem (10). D'Aprile-Mugnai [11] and Coclite [7] proved the existence of a radial positive solution of (10) for $4 \leq p<6$. On the other hand, using a Pohozaev equality, D'Aprile-Mugnai 12 showed that there exists no non-trivial solutions of (10) for $p \leq 2$ or $p \geq 6$. By a new approach, Ruiz [19] fills a gap for the range $2<p<4$. More precisely, he proved the following results:

(i) $(3<p<6$ and $q>0) \exists$ a nontrivial solution, which is a ground state in radial class;

(ii) $(2<p<3$ and small $q>0) \exists$ a nontrivial solution, which is a minimizer of $I_{\infty}$;

(iii) $(2<p \leq 3$ and small $q>0) \exists$ a nontrivial solution emanating from a ground state solution of

$$
-\Delta u+2 m \mu u-|u|^{p-2} u=0 \text { in } \mathbb{R}^{3} ;
$$

(iv) $(2<p \leq 3$ and large $q>0) \nexists$ nontrivial solution of (10).

In 1], Azzollini and Pomponio constructed a ground state solution of (10) for $3<p<6$, which is possibly non-radial. It was shown by Colin and Watanabe 8 that a ground state is unique and radial up to a translation for small $q>0$. This result implies that the solution found by Ruiz coincides with the ground state constructed by Azzollini and Pomponio for small $q>0$ if $3<p<6$. As far as we know, it is unknown whether the ground states is radial when $q>0$ is arbitrary.

Concerning the nonrelativistic limit between solitary waves, one can naturally ask is the following:

Question: For any positive solution $u$ of (10), is there a corresponding family of positive solutions $u_{c}$ of (5), which converges to $u$ as $c \rightarrow \infty$ ?

In this paper, we not only give a complete answer to this question, but also construct blow up solutions to NMKG for $2<p<3$. Our first theorem states the convergence of nonrelativistic limit of ground states between (5) and (10) for $3<p<6$. The theorem contains the existence of a ground state to (5) for $3<p<4$ with arbitrary parameters $m, q, \mu, c>0$ and $c>\sqrt{\mu / m}$, which is not covered by the aforementioned results of Azzollini-Pomponio [2] or Wang [21] (see Proposition 33).

Theorem 1 (Existence and nonrelativistic limit of ground states). Fix arbitrary $\mu, m, q>0$ and $3<p<6$. Then there holds the following:

(i) There exists a ground state solution of (5) for any $c>\sqrt{\mu / m}$.

(ii) Any ground state solution of (5) belongs to $H^{2}\left(\mathbb{R}^{3}\right)$ and converges to a ground state solution of (10) in $H^{2}\left(\mathbb{R}^{3}\right)$ as $c \rightarrow \infty$, up to a translation and a subsequence.

Based on the strategies proposed in [9, 10, we shall prove the convergence of nonrelativistic limit in Theorem 1 by establishing the following steps:

1. Uniform upper estimate of ground energy levels for (5) by the ground energy level for (10), i.e.,

$$
\limsup _{c \rightarrow \infty} E_{c} \leq E_{\infty}
$$

where

$$
E_{c}=\inf \left\{I_{c}(u) \mid u \neq 0, I_{c}^{\prime}(u)=0\right\} \quad \text { and } \quad E_{\infty}=\inf \left\{I_{\infty}(u) \mid u \neq 0, I_{\infty}^{\prime}(u)=0\right\}
$$

2. Uniform $H^{1}$ bounds for ground states $\left\{u_{c}\right\}$ of (5) and solvability of its weak limit $u_{\infty}$ to (10);

3. Energy estimates for establishing $u_{\infty}$ to be a ground state;

4. $H^{1}$ convergence of $u_{c}$ to $u_{\infty}$ and its upgrade to $H^{2}$. 
A new difficulty arises when we prove the step 1 in the case $3<p<4$. It is worth to point out that we couldn't construct a ground state of (5) by using a constrained minimization method for $3<p<4$. It seems not possible to find a suitable constraint working for every admissible parameters $\mu, m, q, c$. As a consequence, we couldn't compare ground states energy levels between (5) and (10). To bypass the obstacle, we directly construct a ground state that satisfies the upper estimate (12). That is, we first show the existence of a family of nontrivial solutions to (5) satisfying the upper estimate (12) by applying a deformation argument developed in [5]. Then, by the compactness of a sequence of solutions to (5), we prove that aforementioned nontrivial solutions to (5) is ground state solutions to (5) (see Proposition [3).

The next theorem covers the case that $2<p<3$ and $q$ is small. We recall the aforementioned results by Ruiz [19, which say the existence of two positive radial solutions $u_{\infty}$ and $v_{\infty}$ of $(10) ; u_{\infty}$ is a perturbation of the ground state to (11) and $v_{\infty}$ is a global minimizer of $I_{\infty}$. In Theorem 2, we show the existence of two radial positive solutions $u_{c}$ and $v_{c}$ to (5) such that $u_{c}$ and $v_{c}$ converges to $u_{\infty}$ and $v_{\infty}$, respectively.

Theorem 2 (Correspondence of two positive solutions for $2<p<3$ ). Assume $2<p<3$. Fix arbitrary but sufficiently small $q>0$ that guarantees the existence of two positive radial solutions $u_{\infty}$ and $v_{\infty}$ to (10) mentioned above. If $c>0$ is sufficiently large, then there exist two distinct radially symmetric positive solutions $u_{c}$ and $v_{c}$ of (5) such that

$$
\text { (i) } \lim _{c \rightarrow \infty}\left\|u_{c}-u_{\infty}\right\|_{H^{1}\left(\mathbb{R}^{3}\right)}=0, \quad \text { (ii) } \lim _{c \rightarrow \infty}\left\|v_{c}-v_{\infty}\right\|_{H^{1}\left(\mathbb{R}^{3}\right)}=0 \text {. }
$$

In [19], Ruiz proved that a global minimizer $v_{\infty}$ of $I_{\infty}$ blows up in $H^{1}$ as $q \rightarrow 0$, which implies that the solution $v_{c}$ constructed in Theorem 2 blows up in $H^{1}$ as $q \rightarrow 0$ and $c \rightarrow \infty$. We point out that Theorem 2 not only proves the correspondence between solitary waves but also establishes a new existence result to (5) for $2<p<3$. As we have seen above, the previous approaches [2, 3, 11, 21] doesn't cover the case that $\omega>0$ is less than but sufficiently close to $\bar{m}$. In this respect, one family of solutions $u_{c}$ is actually not brand new because it is a simple consequence of implicit function theorem, which relies on nondegeneracy of the solution $u_{\infty}$. However, the other family of solutions $v_{c}$ is brand new because $v_{c}$ bifurcates from a global minimizer of $I_{\infty}$, which blows up in $H^{1}$. As for the construction of $v_{c}$, it seems not easy to show whether the global minimum of $I_{c}$ is finite, unlike $I_{\infty}$. This prevents us from simply adopting the minimization argument. To overcome this difficulty, we develop a new deformation argument, which strongly depends on the fact that the global minimum level of $I_{\infty}$ is bounded below. We conjecture that if $c$ is sufficiently large, there exists a global minimizer of $I_{c}$, which converges to $v_{\infty}$.

We organize the paper as follows: In section 2 we give variational settings for NSP and NMKG, and a simple proof for the existence of a ground state to (6) for $3<p<6$. Section 3 is devoted to construct nontrivial solutions to (5) with the energy bound $E_{\infty}$ when $3<p<6$. In Section 4. we prove Theorem 1 by combining the results in Section 3 . In Section 5, we deal with the case $2<p<3$. We construct two radial positive solutions of (5) and prove the convergence of their nonrelativistic limit. Finally, in Appendix, we give basic estimates, which are used in the proofs of main theorems.

Acknowledgement. This research of the second author was supported by Basic Science Research Program through the National Research Foundation of Korea(NRF) funded by the Ministry of Science and ICT (NRF-2020R1C1C1A01006415)

\section{Preliminaries}

This preliminary section introduces basic functional and variational settings for NMKG and NSP. In addition, we provide a simple proof for the existence of a ground state to (6) for every $3<p<6$ and every $e, \bar{m}, \omega>0$ such that $\bar{m}>\omega$.

2.1. Function spaces. The space $D^{1,2}\left(\mathbb{R}^{3}\right)$ is defined by the completion of $C_{0}^{\infty}\left(\mathbb{R}^{3}\right)$ with respect to the norm

$$
\|u\|_{D^{1,2}\left(\mathbb{R}^{3}\right)}=\left(\int_{\Omega}|\nabla u|^{2} d x\right)^{1 / 2} .
$$


For an open set $\Omega \subset \mathbb{R}^{3}$ and $r \in[1, \infty)$, let us denote the norms

$$
\|u\|_{L^{r}(\Omega)}=\left(\int_{\Omega}|u|^{r} d x\right)^{1 / r}, \quad\|u\|_{L^{\infty}(\Omega)}=\underset{x \in \Omega}{\operatorname{ess} \sup }|u(x)|, \quad\|u\|_{H^{1}(\Omega)}=\left(\int_{\Omega}|\nabla u|^{2}+u^{2} d x\right)^{1 / 2} .
$$

We also use the following abbreviations,

$$
\|u\|_{L^{r}}=\|u\|_{L^{r}\left(\mathbb{R}^{3}\right)}, \quad\|u\|_{D^{1,2}}=\|u\|_{D^{1,2}\left(\mathbb{R}^{3}\right)} \quad \text { and } \quad\|u\|_{H^{1}}=\|u\|_{H^{1}\left(\mathbb{R}^{3}\right)} .
$$

We denote by $H_{r}^{1}$ the Sobolev space of radial functions $u$ such that $u, \nabla u$ are in $L^{2}\left(\mathbb{R}^{3}\right)$.

2.2. Variaional settings for NSP. Recall the action functional for (10),

$$
\begin{aligned}
I_{\infty}(u) & =\frac{1}{2} \int_{\mathbb{R}^{3}}|\nabla u|^{2}+2 m \mu u^{2}+\left|\nabla \phi_{u}\right|^{2} d x-\frac{1}{p} \int_{\mathbb{R}^{3}}|u|^{p} d x \\
& =\frac{1}{2} \int_{\mathbb{R}^{3}}|\nabla u|^{2}+2 m \mu u^{2}-q m u^{2} \phi_{u} d x-\frac{1}{p} \int_{\mathbb{R}^{3}}|u|^{p} d x .
\end{aligned}
$$

The map $\lambda: u \in H^{1} \rightarrow \phi_{u} \in D^{1,2}$ is continuously differentiable, where $\phi_{u}$ satisfies (8) (see [11]). Since $\lambda^{\prime}(u)[v]$ satisfies

$$
-\Delta\left(\lambda^{\prime}(u)[v]\right)=-2 q m u v \text { in } \mathbb{R}^{3} \quad \text { for } v \in H^{1},
$$

we have

Then we see that

$$
\int_{\mathbb{R}^{3}} \nabla\left(\lambda^{\prime}(u)[v]\right) \cdot \nabla \phi_{u} d x=-2 q m \int_{\mathbb{R}^{3}} u v \phi_{u} d x
$$

$$
\begin{aligned}
I_{\infty}^{\prime}(u) v & =\int_{\mathbb{R}^{3}} \nabla u \cdot \nabla v+2 m \mu u v+\nabla\left(\lambda^{\prime}(u)[v]\right) \cdot \nabla \phi_{u} d x-\int_{\mathbb{R}^{3}}|u|^{p-2} u v d x \\
& =\int_{\mathbb{R}^{3}} \nabla u \cdot \nabla v+2 m \mu u v-2 q m u v \phi_{u} d x-\int_{\mathbb{R}^{3}}|u|^{p-2} u v d x,
\end{aligned}
$$

which shows that a critical point of $I_{\infty}$ is a weak solution to (10). We define the Nehari and Pohozaev functionals for (10) by

$$
\begin{aligned}
& J_{\infty}(u) \equiv I_{\infty}^{\prime}(u) u=\int_{\mathbb{R}^{3}}|\nabla u|^{2}+2 m \mu u^{2}-2 q m u^{2} \phi_{u}-|u|^{p} d x, \\
& P_{\infty}(u) \equiv \int_{\mathbb{R}^{3}} \frac{1}{2}|\nabla u|^{2}+3 m \mu u^{2}-\frac{5}{2} q m u^{2} \phi_{u}-\frac{3}{p}|u|^{p} d x .
\end{aligned}
$$

We note that the values of $J_{\infty}$ and $P_{\infty}$ should be zero at every critical point of $I_{\infty}$ (see [19]). By defining $G_{\infty}(u) \equiv 2 J_{\infty}(u)-P_{\infty}(u)$, we denote

$$
M_{\infty} \equiv\left\{\left.u \in H^{1} \backslash\{0\}\left|G_{\infty}(u) \equiv \int_{\mathbb{R}^{3}} \frac{3}{2}\right| \nabla u\right|^{2}+m \mu u^{2}-\frac{3}{2} q m u^{2} \phi_{u}-\frac{2 p-3}{p}|u|^{p} d x=0\right\}
$$

and

$$
E_{\infty} \equiv \inf _{u \in M_{\infty}} I_{\infty}(u)
$$

It is proved in [19] that for $3<p<6, E_{\infty}$ equals to the ground energy level for (10), i.e.

$$
E_{\infty}=\inf \left\{I_{\infty}(u) \mid u \neq 0, I_{\infty}^{\prime}(u)=0\right\}
$$

2.3. Variational settings for NMKG. The action functional for (5) is given by

$$
\begin{aligned}
I_{c}(u) & =\frac{1}{2} \int_{\mathbb{R}^{3}}|\nabla u|^{2}+\left(2 m \mu-\frac{\mu^{2}}{c^{2}}\right) u^{2}+\left|\nabla \Phi_{u}\right|^{2}+\left(\frac{q}{c}\right)^{2} u^{2} \Phi_{u}^{2} d x-\frac{1}{p} \int_{\mathbb{R}^{3}}|u|^{p} d x \\
& =\frac{1}{2} \int_{\mathbb{R}^{3}}|\nabla u|^{2}+\left(2 m \mu-\frac{\mu^{2}}{c^{2}}\right) u^{2}-q\left(m-\frac{\mu}{c^{2}}\right) u^{2} \Phi_{u} d x-\frac{1}{p} \int_{\mathbb{R}^{3}}|u|^{p} d x .
\end{aligned}
$$

The map $\Lambda: u \in H^{1} \rightarrow \Phi_{u} \in D^{1,2}$ is continuously differentiable, where $\Phi_{u}$ satisfies (44) (see [11]). For $v \in H^{1}$, since $\Lambda^{\prime}(u)[v]$ satisfies

$$
-\Delta\left(\Lambda^{\prime}(u)[v]\right)+\left(\frac{q}{c}\right)^{2} u^{2}\left(\Lambda^{\prime}(u)[v]\right)=-2\left(\frac{q}{c}\right)^{2} u v \Phi_{u}-2 q\left(m-\frac{\mu}{c^{2}}\right) u v
$$


we have

$$
\int_{\mathbb{R}^{3}} \nabla\left(\Lambda^{\prime}(u)[v]\right) \cdot \nabla \Phi_{u}+\left(\frac{q}{c}\right)^{2} u^{2}\left(\Lambda^{\prime}(u)[v]\right) \Phi_{u} d x=\int_{\mathbb{R}^{3}}-2\left(\frac{q}{c}\right)^{2} u v \Phi_{u}^{2}-2 q\left(m-\frac{\mu}{c^{2}}\right) u v \Phi_{u} d x .
$$

Then we see that for $v \in H^{1}$,

$$
\begin{aligned}
I_{c}^{\prime}(u) v= & \int_{\mathbb{R}^{3}} \nabla u \cdot \nabla v+\left(2 m \mu-\frac{\mu^{2}}{c^{2}}\right) u v+\nabla \Phi_{u} \cdot \nabla\left(\Lambda^{\prime}(u)[v]\right)+\left(\frac{q}{c}\right)^{2} u v \Phi_{u}^{2} \\
& \quad+\left(\frac{q}{c}\right)^{2} u^{2} \Phi_{u}\left(\Lambda^{\prime}(u)[v]\right)-|u|^{p-2} u v d x \\
= & \int_{\mathbb{R}^{3}} \nabla u \cdot \nabla v+\left(2 m \mu-\frac{\mu^{2}}{c^{2}}\right) u v-\left(\frac{q}{c}\right)^{2} u v \Phi_{u}^{2}-2 q\left(m-\frac{\mu}{c^{2}}\right) u v \Phi_{u}-|u|^{p-2} u v d x .
\end{aligned}
$$

In particular, we have

$$
J_{c}(u) \equiv I_{c}^{\prime}(u) u=\int_{\mathbb{R}^{3}}|\nabla u|^{2}+\left(2 m \mu-\frac{\mu^{2}}{c^{2}}\right) u^{2}-\left(\frac{q}{c}\right)^{2} u^{2} \Phi_{u}^{2}-2 q\left(m-\frac{\mu}{c^{2}}\right) u^{2} \Phi_{u}-|u|^{p} d x .
$$

For any critical point $w_{c}$ of $I_{c}$, it is clear that $J_{c}\left(w_{c}\right)=0$ and it is shown in 12 that the Pohozaev's identity $P_{c}\left(w_{c}\right)=0$ holds true, where

$$
P_{c}(u) \equiv \int_{\mathbb{R}^{3}} \frac{1}{2}|\nabla u|^{2}+\frac{3}{2}\left(2 m \mu-\frac{\mu^{2}}{c^{2}}\right) u^{2}-\frac{q^{2}}{c^{2}} \Phi_{u}^{2} u^{2}-\frac{5}{2} q\left(m-\frac{\mu}{c^{2}}\right) u^{2} \Phi_{u}-\frac{3}{p}|u|^{p} d x .
$$

2.4. Existence of a ground state for $3<p<6$. We recall the equation (6), written as

$$
-\Delta u+\left(\bar{m}^{2}-\left(e \varphi_{u}+\omega\right)^{2}\right) u=|u|^{p-2} u \text { in } \mathbb{R}^{3}
$$

where $e>0,0<\omega<\bar{m}$ and $\varphi_{u}$ is a unique solution of

$$
-\Delta \varphi+e^{2} \varphi u^{2}=-e \omega u^{2} .
$$

Here we point out that by the maximum principle, we have the uniform bound

$$
-\frac{\omega}{e} \leq \varphi_{u} \leq 0
$$

Proposition 3. Assume that $3<p<6, e>0$ and $0<\omega<\bar{m}$. If there exists a non-trivial solution of (14), then there exists a non-trivial ground state solution of (14).

Proof. Suppose that there exists a non-trivial solution solution of (14). We recall the action functional of (14)

$$
I(u)=\frac{1}{2} \int_{\mathbb{R}^{3}}|\nabla u|^{2}+\left(\bar{m}^{2}-\omega^{2}\right) u^{2}-e \omega \varphi_{u} u^{2} d x-\frac{1}{p} \int_{\mathbb{R}^{3}}|u|^{p} d x .
$$

and consider the minimization problem

$$
\mathcal{S}=\inf \{I(u) \mid u \in \mathcal{B}\},
$$

where

$$
\mathcal{B} \equiv\left\{u \in H^{1} \mid u \text { is a non-trivial solution solution of (14) }\right\} .
$$

By the definition, a ground state solution $u$ of (14) is a nontrivial critical point of $I$ satisfying $I(u)=\mathcal{S}$. Let us define

$$
\left\{\begin{array}{l}
T(u):=I^{\prime}(u) u=\int_{\mathbb{R}^{3}}|\nabla u|^{2}+\left(\bar{m}^{2}-\omega^{2}\right) u^{2}-2 e \omega \varphi_{u} u^{2}-e^{2} \varphi_{u}^{2} u^{2}-|u|^{p} d x \\
Q(u):=\int_{\mathbb{R}^{3}} \frac{1}{2}|\nabla u|^{2}+\frac{3}{2}\left(\bar{m}^{2}-\omega^{2}\right) u^{2}-\frac{5}{2} e \omega \varphi_{u} u^{2}-e^{2} \varphi_{u}^{2} u^{2}-\frac{3}{p}|u|^{p} d x .
\end{array}\right.
$$

Since $T(v)=Q(v)=0$ for any $v \in \mathcal{B}$, (see [12]), one has

$$
\begin{aligned}
\frac{5 p-12}{2} I(v) & =\frac{5 p-12}{2} I(v)-T(v)+\frac{4-p}{2} Q(v) \\
& =\int_{\mathbb{R}^{3}}(p-3)|\nabla v|^{2}+\frac{p-2}{2}\left(\bar{m}^{2}-\omega^{2}\right) v^{2}+\frac{p-2}{2} e^{2} v^{2} \varphi_{v}^{2} d x
\end{aligned}
$$

for $v \in \mathcal{B}$. This implies that $\mathcal{S} \geq 0$. 
Let $\left\{u_{n}\right\}$ be a minimizing sequence of $\mathcal{S}$. From the estimates

$$
\frac{5 p-12}{2} \mathcal{S}+o(1)=\int_{\mathbb{R}^{3}}(p-3)\left|\nabla u_{n}\right|^{2}+\frac{p-2}{2}\left(\bar{m}^{2}-\omega^{2}\right) u_{n}^{2}+\frac{p-2}{2} e^{2} u_{n}^{2} \varphi_{u_{n}}^{2} d x
$$

and

$$
\begin{aligned}
0=T\left(u_{n}\right) & =\int_{\mathbb{R}^{3}}\left|\nabla u_{n}\right|^{2}+\left(\bar{m}^{2}-\omega^{2}\right) u_{n}^{2}-\varphi_{u_{n}}\left(2 e \omega+e^{2} \varphi_{u_{n}}\right) u_{n}^{2}-\left|u_{n}\right|^{p} d x \\
& \geq \int_{\mathbb{R}^{3}}\left|\nabla u_{n}\right|^{2}+\left(\bar{m}^{2}-\omega^{2}\right) u_{n}^{2}-\left|u_{n}\right|^{p} d x \geq C\left\|u_{n}\right\|_{L^{p}}^{2 / p}-\left\|u_{n}\right\|_{L^{p}}^{p},
\end{aligned}
$$

we deduce that $\left(u_{n}\right)$ is bounded in $H^{1}$ and $\left\|u_{n}\right\|_{L^{p}} \geq C_{1}$ for some positive constant $C_{1}$. Then we see from Lemma 1.1 in [13,

$$
\sup _{x \in \mathbb{R}^{3}} \int_{B_{1}(x)}\left|u_{n}\right|^{2} d x=\int_{B_{1}\left(x_{n}\right)}\left|u_{n}\right|^{2} d x \geq C_{2}>0
$$

where $x_{n} \in \mathbb{R}^{3}$ and $C_{2}$ is a positive constant. Then we may assume that $u_{n}\left(\cdot+x_{n}\right)$ converges to $u \not \equiv 0$ weakly in $H^{1}$. It is standard to show that $u$ is a non-trivial critical point of $I$. Moreover, by (15) and the fact that $u$ is a non-trivial critical point of $I$, we see that

$$
\begin{aligned}
\frac{5 p-12}{2} \mathcal{S} & =\liminf _{n \rightarrow \infty} \int_{\mathbb{R}^{3}}(p-3)\left|\nabla u_{n}\right|^{2}+\frac{p-2}{2}\left(\bar{m}^{2}-\omega^{2}\right) u_{n}^{2}+\frac{p-2}{2} e^{2} u_{n}^{2} \varphi_{u_{n}}^{2} d x \\
& \geq \int_{\mathbb{R}^{3}}(p-3)|\nabla u|^{2}+\frac{p-2}{2}\left(\bar{m}^{2}-\omega^{2}\right) u^{2}+\frac{p-2}{2} e^{2} u^{2} \varphi_{u}^{2} d x=\frac{5 p-12}{2} I(u),
\end{aligned}
$$

which implies that $u$ is a non-trivial ground state solution of (14).

Observe that Proposition 3 implies the existence of a ground state to (14) for any $e, \bar{m}, \omega>0$ such that $0<\omega<\bar{m}$ since there exists a nontrivial solution at those ranges of parameters by [3].

\section{Construction of nontrivial solutions to NKGM with the Energy bound $E_{\infty}$}

In this section, based on the idea of [5], we shall construct a family of nontrivial solutions $w_{c}$ to (5) satisfying

$$
\limsup _{c \rightarrow \infty} I_{c}\left(w_{c}\right) \leq E_{\infty} .
$$

Before proceeding further, we first introduce a modified functional $\tilde{I}_{c}$ as

$$
\tilde{I}_{c}(u)=\frac{1}{2} \int_{\mathbb{R}^{3}}|\nabla u|^{2}+\left(2 m \mu-\frac{\mu^{2}}{c^{2}}\right) u^{2}-q\left(m-\frac{\mu}{c^{2}}\right) u^{2} \Phi_{u} d x-\frac{1}{p} \int_{\mathbb{R}^{3}} u_{+}^{p} d x,
$$

where $c>0$ and $u_{+}=\max \{u, 0\}$. A critical point of $\tilde{I}_{c}$ corresponds to a solution of

$$
\begin{aligned}
&-\Delta u+\left(2 m \mu-\left(\frac{\mu}{c}\right)^{2}\right) u-\left(\frac{q}{c}\right)^{2} u \Phi^{2}-2 q\left(m-\frac{\mu}{c^{2}}\right) u \Phi-u_{+}^{p-1}=0 \text { in } \mathbb{R}^{3}, \\
&-\Delta \Phi+\frac{q^{2}}{c^{2}} u^{2} \Phi=-q\left(m-\frac{\mu}{c^{2}}\right) u^{2} \text { in } \mathbb{R}^{3} .
\end{aligned}
$$

It is possible to show from the maximum principle that a critical point $u$ of $\tilde{I}_{c}$ is positive everywhere in $\mathbb{R}^{3}$ for $c \geq \sqrt{\frac{2 m}{\mu}}$. Indeed, since $-\frac{c^{2}}{q}\left(m-\frac{\mu}{c^{2}}\right) \leq \Phi_{u} \leq 0$, multiplying $u_{-}$to the equation

$$
-\Delta u+\left(2 m \mu-\left(\frac{\mu}{c}\right)^{2}\right) u-\left(\frac{q}{c}\right)^{2} u \Phi_{u}^{2}-2 q\left(m-\frac{\mu}{c^{2}}\right) u \Phi_{u}-u_{+}^{p-1}=0 \text { in } \mathbb{R}^{3}
$$

and then integrating over $\mathbb{R}^{3}$, we have

$$
\begin{aligned}
& \int_{\mathbb{R}^{3}}\left|\nabla u_{-}\right|^{2}+\left(2 m \mu-\frac{\mu^{2}}{c^{2}}\right) u_{-}^{2} d x \\
& \leq \int_{\mathbb{R}^{3}}\left|\nabla u_{-}\right|^{2}+\left(2 m \mu-\frac{\mu^{2}}{c^{2}}\right) u_{-}^{2}-u_{-}^{2} \Phi_{u}\left[\left(\frac{q}{c}\right)^{2} \Phi_{u}+2 q\left(m-\frac{\mu}{c^{2}}\right)\right] d x=0
\end{aligned}
$$


where $u_{-}=\min \{u, 0\}$. Therefore a nontrivial critical point of $\tilde{I}_{c}$ gives a positive solution to (5). We also define

$$
\begin{aligned}
\tilde{I}_{\infty}(u) & :=\frac{1}{2} \int_{\mathbb{R}^{3}}|\nabla u|^{2}+2 m \mu u^{2}-q m u^{2} \phi_{u} d x-\frac{1}{p} \int_{\mathbb{R}^{3}} u_{+}^{p} d x, \\
\tilde{J}_{\infty}(u) & :=I_{\infty}^{\prime}(u) u=\int_{\mathbb{R}^{3}}|\nabla u|^{2}+2 m \mu u^{2}-2 q m u^{2} \phi_{u}-u_{+}^{p} d x, \\
\tilde{P}_{\infty}(u) & :=\int_{\mathbb{R}^{3}} \frac{1}{2}|\nabla u|^{2}+3 m \mu u^{2}-\frac{5}{2} q m u^{2} \phi_{u}-\frac{3}{p} u_{+}^{p} d x .
\end{aligned}
$$

Let $\mathcal{A} \equiv\left\{u \in H^{1} \mid \tilde{I}_{\infty}^{\prime}(u)=0, \tilde{I}_{\infty}(u)=E_{\infty}\right.$, and $\left.\max _{\mathbb{R}^{3}} u=u(0)\right\}$. We note that $\mathcal{A} \neq \emptyset$. Indeed, if $u \in \mathcal{M}_{\infty}$ satisfies $I_{\infty}(u)=E_{\infty}$, we see that $|u|$ satisfies $\tilde{I}_{\infty}(|u|)=E_{\infty}$ and $\tilde{I}_{\infty}^{\prime}(|u|)=0$.

Proposition 4. For $3<p<6$, there exist positive constants $C_{1}$ and $C_{2}$ independent of $U \in \mathcal{A}$ such that for $U \in \mathcal{A}$,

$$
U(x)+|\nabla U(x)| \leq C_{1} \exp \left(-C_{2}|x|\right) .
$$

Moreover, $\inf _{U \in \mathcal{A}}\|U\|_{L^{\infty}}>0$.

Proof. Let $U \in \mathcal{A}$. It follows from

$$
\begin{aligned}
E_{\infty} & =\tilde{I}_{\infty}(U)=\tilde{I}_{\infty}(U)-\frac{2}{5 p-12} \tilde{J}_{\infty}(U)-\frac{p-4}{5 p-12} \tilde{P}_{\infty}(U) \\
& =\int_{\mathbb{R}^{3}} \frac{2(p-3)}{5 p-12}|\nabla U|^{2}+\frac{2(p-2)}{5 p-12} m \mu U^{2} d x
\end{aligned}
$$

where $U \in \mathcal{A}$, that $\mathcal{A}$ is bounded in $H^{1}$ if $3<p<6$. Then, since

$$
\begin{aligned}
\left\|\phi_{U}+|U|^{p-2}\right\|_{L^{\frac{6}{p-2}}(\Omega)} & \leq\left\|\phi_{U}\right\|_{L^{\frac{6}{p-2}}(\Omega)}+\|U\|_{L^{6}(\Omega)}^{p-2} \leq|\Omega|^{\frac{p-2}{6}-\frac{1}{6}}\left\|\phi_{U}\right\|_{L^{6}(\Omega)}+\|U\|_{L^{6}(\Omega)}^{p-2} \\
& \leq C\left(|\Omega|^{\frac{p-2}{6}-\frac{1}{6}}\|U\|_{H^{1}}^{2}+\|U\|_{H^{1}}^{p-2}\right),
\end{aligned}
$$

where $3<p<6, U \in \mathcal{A}, \Omega$ is a bounded domain in $\mathbb{R}^{3}$ and $C$ is a positive constant independent of $U \in \mathcal{A}$, we see that $\mathcal{A}$ is bounded in $L^{\infty}$ (see [20, Theorem 4.1]).

We claim that $\lim _{|x| \rightarrow \infty} U(x)=0$ uniformly for $U \in \mathcal{A}$. Indeed, contrary to our claim, suppose that there exist $\left\{U_{i}\right\}_{i=1}^{\infty} \subset \mathcal{A}$ and $\left\{x_{i}\right\}_{i=1}^{\infty} \subset \mathbb{R}^{N}$ satisfying $\lim _{i \rightarrow \infty}\left|x_{i}\right|=\infty$ and $\liminf _{i \rightarrow \infty} U_{i}\left(x_{i}\right)>$ 0 . Denote $V_{i} \equiv U_{i}\left(\cdot+x_{i}\right)$. We note that if $u_{i} \rightarrow u$ in $H^{1}, \phi_{u_{i}} \rightarrow \phi_{u}$ in $D^{1,2}$. Then if $u_{i} \rightarrow u$ in $H^{1}$, for $\psi \in C_{0}^{\infty}\left(\mathbb{R}^{3}\right)$,

$$
\int_{\mathbb{R}^{3}}\left(u_{i} \phi_{u_{i}}-u \phi_{u}\right) \psi d x=\int_{\mathbb{R}^{3}}\left(u_{i}-u\right) \phi_{u_{i}} \psi+u\left(\phi_{u_{i}}-\phi_{u}\right) \psi d x=o(1)
$$

as $i \rightarrow \infty$. By (18) and the fact that $\left\{U_{i}, V_{i}\right\}_{i=1}^{\infty}$ is bounded in $H^{1}$, we see that $U_{i}$ and $V_{i}$ converge to $U$ and $V$ weakly in $H^{1}$ as $i \rightarrow \infty$, up to a subsequence, respectively, where $U$ and $V$ are non-trivial solutions of (10). It follows from (17) that for $2 R \leq\left|x_{i}\right|$,

$$
\begin{aligned}
E_{\infty}= & \liminf _{i \rightarrow \infty} \tilde{I}_{\infty}\left(U_{i}\right)=\liminf _{i \rightarrow \infty} \int_{\mathbb{R}^{3}} \frac{2(p-3)}{5 p-12}\left|\nabla U_{i}\right|^{2}+\frac{2(p-2)}{5 p-12} m \mu U_{i}^{2} d x \\
\geq & \liminf _{i \rightarrow \infty} \int_{B(0, R)} \frac{2(p-3)}{5 p-12}\left|\nabla U_{i}\right|^{2}+\frac{2(p-2)}{5 p-12} m \mu U_{i}^{2} d x \\
& \quad+\liminf _{i \rightarrow \infty} \int_{B\left(x_{i}, R\right)} \frac{2(p-3)}{5 p-12}\left|\nabla U_{i}\right|^{2}+\frac{2(p-2)}{5 p-12} m \mu U_{i}^{2} d x \\
\geq & \int_{B(0, R)} \frac{2(p-3)}{5 p-12}|\nabla U|^{2}+\frac{2(p-2)}{5 p-12} m \mu U^{2} d x \\
& \quad+\int_{B(0, R)} \frac{2(p-3)}{5 p-12}|\nabla V|^{2}+\frac{2(p-2)}{5 p-12} m \mu V^{2} d x .
\end{aligned}
$$

Since

$$
\tilde{I}_{\infty}(U), \tilde{I}_{\infty}(V) \geq \tilde{I}_{\infty}(W) \text { for any } W \in \mathcal{A}
$$


if we take large $R>0$ in (19), we deduce a contradiction. This implies that $\lim _{|x| \rightarrow \infty} U(x)=0$ uniformly for $U \in \mathcal{A}$.

We note that for large $|x|$,

$$
\begin{aligned}
\phi_{U}(x) & =-\frac{q m}{4 \pi} \int_{\mathbb{R}^{3}} \frac{U^{2}(y)}{|x-y|} d y=-\frac{q m}{4 \pi} \int_{B(x, R)} \frac{U^{2}(y)}{|x-y|} d y-\frac{q m}{4 \pi} \int_{\mathbb{R}^{3} \backslash B(x, R)} \frac{U^{2}(y)}{|x-y|} d y \\
& =o(1) R^{2}+O(1) \frac{1}{R}=o(1)
\end{aligned}
$$

uniformly in $U \in \mathcal{A}$. Then, by the comparison principle and the elliptic estimates, we see that for $U \in \mathcal{A}$,

$$
U(x)+|\nabla U(x)| \leq C_{1} \exp \left(-C_{2}|x|\right)
$$

where $C_{1}$ and $C_{2}$ are positive constants independent of $U \in \mathcal{A}$.

To show $\inf _{U \in \mathcal{A}}\|U\|_{L^{\infty}}>0$, we assume that there exists $\left\{U_{i}\right\}_{i=1}^{\infty} \subset \mathcal{A}$ such that $\left\|U_{i}\right\|_{L^{\infty}} \rightarrow 0$ as $i \rightarrow \infty$. Then, since $U_{i}$ satisfies

$$
-\Delta U_{i}+2 m \mu U_{i}-U_{i}^{p-1} \leq-\Delta U_{i}+2 m \mu U_{i}-2 q m U_{i} \phi_{U_{i}}-U_{i}^{p-1}=0 \text { in } \mathbb{R}^{3},
$$

we see that $\left\|U_{i}\right\|_{H^{1}} \rightarrow 0$ as $i \rightarrow \infty$, which is a contradiction to (17).

For a fixed $U_{0} \in \mathcal{A}$, we define $\gamma(t)(x)=t^{2} U_{0}(t x)$. It follows from

$$
\tilde{I}_{\infty}(\gamma(t))=\frac{1}{2} \int_{\mathbb{R}^{3}} t^{3}\left|\nabla U_{0}\right|^{2}+2 m \mu t U_{0}^{2}-q m t^{3} U_{0}^{2} \phi_{U_{0}} d x-\frac{t^{2 p-3}}{p} \int_{\mathbb{R}^{3}} U_{0}^{p} d x
$$

that for $3<p<6$, there exists $t_{0}>1$ such that $\tilde{I}_{\infty}(\gamma(t))<0$ for $t \geq t_{0}$. Moreover, by [19, Lemma 3.3 and the fact that $U_{0}$ is a critical point of $\tilde{I}_{\infty}$, we see that for $3<p<6, t=1$ is a unique critical point of $\tilde{I}_{\infty}(\gamma(t))$, corresponding to its maximum.

We define

$$
\hat{e}_{c}:=\max _{t \in\left[0, t_{0}\right]} \tilde{I}_{c}(\gamma(t)), \quad \text { and } \quad e_{c}:=\inf _{\Gamma \in \mathcal{W}} \max _{s \in[0,1]} \tilde{I}_{c}(\Gamma(s)),
$$

where $\mathcal{W} \equiv\left\{\Gamma \in C\left([0,1], H^{1}\right) \mid \Gamma(0)=0, \Gamma(1)=\gamma\left(t_{0}\right)\right\}$.

Proposition 5. Let $3<p<6$. Then we have

$$
\limsup _{c \rightarrow \infty} \hat{e}_{c} \leq E_{\infty}
$$

Proof. We see from Lemma 21 and the scaling $\phi_{t^{2} U_{0}(t \cdot)}=t^{2} \phi_{U_{0}}(t \cdot)$, that for $t \in\left[0, t_{0}\right]$,

$$
\begin{aligned}
\tilde{I}_{c}(\gamma(t))= & \frac{1}{2} \int_{\mathbb{R}^{3}}\left|t^{3}\left(\nabla U_{0}\right)(t x)\right|^{2}+\left(2 m \mu-\frac{\mu^{2}}{c^{2}}\right) t^{4} U_{0}^{2}(t x)-q\left(m-\frac{\mu}{c^{2}}\right) t^{4} U_{0}^{2}(t x) \Phi_{t^{2} U_{0}(t \cdot)} d x \\
& \quad-\frac{t^{2 p}}{p} \int_{\mathbb{R}^{3}}\left(U_{0}(t x)\right)^{p} d x \\
= & \frac{1}{2} \int_{\mathbb{R}^{3}}\left|t^{3}\left(\nabla U_{0}\right)(t x)\right|^{2}+2 m \mu t^{4} U_{0}^{2}(t x)-q m t^{4} U_{0}^{2}(t x) \phi_{t^{2} U_{0}(t \cdot)} d x \\
& \quad-\frac{t^{2 p}}{p} \int_{\mathbb{R}^{3}}\left(U_{0}(t x)\right)^{p} d x+o(1) \\
= & \frac{1}{2} \int_{\mathbb{R}^{3}} t^{3}\left|\nabla U_{0}\right|^{2}+2 m \mu t U_{0}^{2}-q m t^{3} U_{0}^{2} \phi_{U_{0}} d x-\frac{t^{2 p-3}}{p} \int_{\mathbb{R}^{3}}\left(U_{0}\right)^{p} d x+o(1) \\
= & \tilde{I}_{\infty}(\gamma(t))+o(1),
\end{aligned}
$$

where $o(1)$ is uniform in $t \in\left[0, t_{0}\right]$ as $c \rightarrow \infty$. Thus, since $t=1$ is a unique maximum point of $\tilde{I}_{\infty}(\gamma(t))$ for $3<p<6$, we deduce that

$$
\hat{e}_{c}=\max _{s \in[0,1]} \tilde{I}_{c}\left(\gamma\left(t_{0} s\right)\right)=\tilde{I}_{\infty}\left(U_{0}\right)+o(1)=E_{\infty}+o(1)
$$

as $c \rightarrow \infty$. 
Proposition 6. Let $3<p<6$. Then we have

$$
\liminf _{c \rightarrow \infty} e_{c} \geq E_{\infty}
$$

Proof. We note that for $\Gamma \in \mathcal{W}$,

$$
\begin{aligned}
\tilde{I}_{c}(\Gamma(t))= & \frac{1}{2} \int_{\mathbb{R}^{3}}|\nabla \Gamma(t)|^{2}+2 m \mu \Gamma^{2}(t)-q m \Gamma^{2}(t) \phi_{\Gamma(t)} d x-\frac{1}{p} \int_{\mathbb{R}^{3}}(\Gamma(t))_{+}^{p} d x \\
& \quad-\frac{1}{c^{2}} \int_{\mathbb{R}^{3}} \mu^{2} \Gamma^{2}(t)-q \mu \Gamma^{2}(t) \Phi_{\Gamma(t)} d x-\frac{1}{2} q m \int_{\mathbb{R}^{3}} \Gamma^{2}(t)\left(\Phi_{\Gamma(t)}-\phi_{\Gamma(t)}\right) d x \\
= & \tilde{I}_{\infty}(\Gamma(t))+G_{c}(t),
\end{aligned}
$$

where $G_{c}(t) \equiv-\frac{1}{c^{2}} \int_{\mathbb{R}^{3}} \mu^{2} \Gamma^{2}(t)-q \mu \Gamma^{2}(t) \Phi_{\Gamma(t)} d x-\frac{1}{2} q m \int_{\mathbb{R}^{3}} \Gamma^{2}(t)\left(\Phi_{\Gamma(t)}-\phi_{\Gamma(t)}\right) d x$. By Lemma 21 we have

$$
\left|G_{c}(t)\right|=o(1) \text { uniformly in } t \in[0,1] \text { as } c \rightarrow \infty \text {. }
$$

Then, since

$$
\max _{t \in[0,1]} \tilde{I}_{\infty}(\Gamma(t)) \geq E_{\infty}
$$

where $\Gamma \in \mathcal{W}$ (see [1, Lemma 2.4]), we have

$$
e_{c} \geq E_{\infty}+\inf _{\Gamma \in \mathcal{W}} \max _{t \in[0,1]} G_{c}(t) \geq E_{\infty}-\inf _{\Gamma \in \mathcal{W}} \max _{t \in[0,1]}\left|G_{c}(t)\right|=E_{\infty}+o(1)
$$

as $c \rightarrow \infty$.

We define

$$
\mathcal{X} \equiv\left\{U(\cdot-y) \mid U \in \mathcal{A}, y \in \mathbb{R}^{3}\right\}
$$

and

$$
N_{d}(\mathcal{X}) \equiv\left\{u \in H^{1} \mid \inf _{v \in \mathcal{X}}\|u-v\|_{H^{1}} \leq d\right\}
$$

where $d>0$ is a constant and $\mathcal{A} \equiv\left\{u \in H^{1} \mid \tilde{I}_{\infty}^{\prime}(u)=0, \tilde{I}_{\infty}(u)=E_{\infty}\right.$, and $\left.\max _{\mathbb{R}^{3}} u=u(0)\right\}$.

Proposition 7. Let $3<p<6$. For large $c>0$, for small $d>0$, and for any $d^{\prime} \in(0, d)$, there exists $\nu \equiv \nu\left(d, d^{\prime}\right)>0$ independent of $c>0$ such that

$$
\inf \left\{\left\|\tilde{I}_{c}^{\prime}(u)\right\|_{H^{-1}} \mid \tilde{I}_{c}(u) \leq \hat{e}_{c}, u \in N_{d}(\mathcal{X}) \backslash N_{d^{\prime}}(\mathcal{X})\right\} \geq \nu>0
$$

Proof. Let $\left\{c_{i}\right\}_{i=1}^{\infty}$ be such that $\lim _{i \rightarrow \infty} c_{i}=\infty$. It suffices to show that for small $d>0$, if

$$
u_{c_{i}} \in N_{d}(\mathcal{X}), \quad \tilde{I}_{c_{i}}\left(u_{c_{i}}\right) \leq \hat{e}_{c_{i}}, \quad \text { and }\left\|\tilde{I}_{c_{i}}^{\prime}\left(u_{c_{i}}\right)\right\|_{H^{-1}} \rightarrow 0
$$

as $i \rightarrow \infty$, then

$$
\inf _{v \in \mathcal{X}}\left\|u_{c_{i}}-v\right\|_{H^{1}} \rightarrow 0 \text { as } i \rightarrow \infty \text {. }
$$

For the sake of simplicity of notation, we write $c$ for $c_{i}$. Since $u_{c} \in N_{d}(\mathcal{X})$, we have

$$
\left\|u_{c}(x)-U_{c}\left(x-y_{c}\right)\right\|_{H^{1}} \leq d,
$$

where $U_{c} \in \mathcal{A}$ and $y_{c} \in \mathbb{R}^{3}$. We define $\eta \in C_{0}^{\infty}\left(\mathbb{R}^{3}\right)$ such that $0 \leq \eta \leq 1, \eta(x)=1$ for $|x| \leq 1$, $\eta(x)=0$ for $|x| \geq 2$, and $|\nabla \eta| \leq 2$. Also, we set $\tilde{\eta}_{c}(x)=\eta\left(\frac{x-y_{c}}{c}\right)$. We divide the proof into three steps.

Step 1. $\tilde{I}_{c}\left(u_{c}\right) \geq \tilde{I}_{\infty}\left(v_{c}\right)+\tilde{I}_{\infty}\left(w_{c}\right)+o(1)$ as $c \rightarrow \infty$, where $v_{c}=\tilde{\eta}_{c} u_{c}$ and $w_{c}=\left(1-\tilde{\eta}_{c}\right) u_{c}$.

We claim first that for $\alpha \in(2,6)$,

$$
\lim _{c \rightarrow \infty} \int_{B\left(y_{c}, 2 c\right) \backslash B\left(y_{c}, c\right)}\left|u_{c}\right|^{\alpha} d x=0 .
$$

Suppose that there exist $z_{c} \in B\left(y_{c}, 2 c\right) \backslash B\left(y_{c}, c\right)$ and $R>0$ such that

$$
\liminf _{c \rightarrow \infty} \int_{B\left(z_{c}, R\right)}\left|u_{c}\right|^{2} d x>0 .
$$


Denote $\tilde{u}_{c}=u_{c}\left(\cdot+z_{c}\right)$. We note that, by Lemma 21 and the fact that $\left\|u_{c}\right\|_{H^{1}}$ is bounded, for $\psi \in C_{0}^{\infty}\left(\mathbb{R}^{3}\right)$,

$$
\begin{aligned}
& \tilde{I}_{c}^{\prime}\left(\tilde{u}_{c}\right) \psi \\
& =\int_{\mathbb{R}^{3}} \nabla \tilde{u}_{c} \cdot \nabla \psi+\left(2 m \mu-\frac{\mu^{2}}{c^{2}}\right) \tilde{u}_{c} \psi-\left(\frac{q}{c}\right)^{2} \tilde{u}_{c} \psi \Phi_{\tilde{u}_{c}}^{2}-2 q\left(m-\frac{\mu}{c^{2}}\right) \tilde{u}_{c} \psi \Phi_{\tilde{u}_{c}}-\left(\tilde{u}_{c}\right)_{+}^{p-1} \psi d x \\
& =\int_{\mathbb{R}^{3}} \nabla \tilde{u}_{c} \cdot \nabla \psi+2 m \mu \tilde{u}_{c} \psi-2 q m \tilde{u}_{c} \psi \phi_{\tilde{u}_{c}}-\left(\tilde{u}_{c}\right)_{+}^{p-1} \psi d x \\
& \quad+\int_{\mathbb{R}^{3}}-\frac{\mu^{2}}{c^{2}} \tilde{u}_{c} \psi-\left(\frac{q}{c}\right)^{2} \tilde{u}_{c} \psi \Phi_{\tilde{u}_{c}}^{2}+2 q \frac{\mu}{c^{2}} \tilde{u}_{c} \psi \Phi_{\tilde{u}_{c}}-2 q m \tilde{u}_{c} \psi\left(\Phi_{\tilde{u}_{c}}-\phi_{\tilde{u}_{c}}\right) d x \\
& =\tilde{I}_{\infty}^{\prime}\left(\tilde{u}_{c}\right) \psi+o(1)
\end{aligned}
$$

as $c \rightarrow \infty$. By (18) and the assumption that $\left\|\tilde{I}_{c}^{\prime}\left(u_{c}\right)\right\|_{H^{-1}} \rightarrow 0$ as $c \rightarrow \infty$, we have $u_{c}\left(\cdot+z_{c}\right) \rightarrow \tilde{U} \not \equiv 0$ in $H^{1}$, where $\tilde{U}$ satisfies $\tilde{I}_{\infty}^{\prime}(\tilde{U})=0$. By (17), we have

$$
\int_{\mathbb{R}^{3}}|\nabla \tilde{U}|^{2}+\tilde{U} d x \geq E_{\infty}\left(\max \left\{\frac{2(p-3)}{5 p-12}, \frac{2(p-2)}{5 p-12} m \mu\right\}\right)^{-1}
$$

Then, by Proposition 4 and the fact that $\left|z_{c}-y_{c}\right| \geq c$, we see that for $R>0$,

$$
\begin{aligned}
d^{2} & \geq\left\|u_{c}(x)-U_{c}\left(x-y_{c}\right)\right\|_{H^{1}}^{2}=\left\|\tilde{u}_{c}(x)-U_{c}\left(x+z_{c}-y_{c}\right)\right\|_{H^{1}}^{2} \\
& \geq\left\|\tilde{u}_{c}(x)-U_{c}\left(x+z_{c}-y_{c}\right)\right\|_{H^{1}(B(0, R))}^{2}=\left\|\tilde{u}_{c}(x)\right\|_{H^{1}(B(0, R))}^{2}+o(1) \geq\|\tilde{U}\|_{H^{1}(B(0, R))}^{2}
\end{aligned}
$$

as $c \rightarrow \infty$. If we take small $d>0$, by (25), we deduce a contradiction. Since there does not exists such a sequence $\left\{z_{c}\right\}$ satisfying (23), by [13, Lemma 1.1], we deduce (22). Then, by (22), we have

$$
\int_{\mathbb{R}^{3}}\left(u_{c}\right)_{+}^{p}-\left(v_{c}\right)_{+}^{p}-\left(w_{c}\right)_{+}^{p} d x=o(1)
$$

as $c \rightarrow \infty$, where $v_{c}$ and $w_{c}$ are given in (22) above. By (22) and Lemma 17.

$$
\begin{aligned}
\int_{B\left(y_{c}, 2 c\right) \backslash B\left(y_{c}, c\right)} u_{c}^{2}\left|\phi_{u_{c}}\right| d x & \leq\left\|\phi_{u_{c}}\right\|_{L^{6}\left(B\left(y_{c}, 2 c\right) \backslash B\left(y_{c}, c\right)\right)}\left\|u_{c}^{2}\right\|_{L^{6 / 5}\left(B\left(y_{c}, 2 c\right) \backslash B\left(y_{c}, c\right)\right)} \\
& \leq C_{1}\left\|u_{c}\right\|_{H^{1}}^{2}\left\|u_{c}\right\|_{L^{12 / 5}\left(B\left(y_{c}, 2 c\right) \backslash B\left(y_{c}, c\right)\right)}^{2} \rightarrow 0
\end{aligned}
$$

as $c \rightarrow \infty$, where $C_{1}$ is a positive constant. From this and the fact that $\left|\nabla \eta_{c}\right| \leq 2 / c$, we see that

$$
\begin{aligned}
& \int_{\mathbb{R}^{3}} v_{c}^{2} \phi_{v_{c}}+w_{c}^{2} \phi_{w_{c}}-u_{c}^{2} \phi_{u_{c}} d x \\
& =\int_{B\left(y_{c}, c\right) \cup\left(\mathbb{R}^{3} \backslash B\left(y_{c}, 2 c\right)\right)} v_{c}^{2} \phi_{v_{c}}+w_{c}^{2} \phi_{w_{c}}-u_{c}^{2} \phi_{u_{c}} d x+o(1) \\
& =\frac{q m}{4 \pi} \int_{B\left(y_{c}, c\right) \cup\left(\mathbb{R}^{3} \backslash B\left(y_{c}, 2 c\right)\right)} \int_{\mathbb{R}^{3}} \frac{u_{c}^{2}(x) u_{c}^{2}(y)-v_{c}^{2}(x) v_{c}^{2}(y)-w_{c}^{2}(x) w_{c}^{2}(y)}{|x-y|} d y d x+o(1) \\
& =\frac{q m}{4 \pi} \int_{B\left(y_{c}, c\right)} \int_{\mathbb{R}^{3}} \frac{u_{c}^{2}(x)\left(u_{c}^{2}(y)-v_{c}^{2}(y)\right)}{|x-y|} d y d x \\
& \quad+\frac{q m}{4 \pi} \int_{\mathbb{R}^{3} \backslash B\left(y_{c}, 2 c\right)} \int_{\mathbb{R}^{3}} \frac{u_{c}^{2}(x)\left(u_{c}^{2}(y)-w_{c}^{2}(y)\right)}{|x-y|} d y d x+o(1) \geq o(1)
\end{aligned}
$$


as $c \rightarrow \infty$. Thus, by (26), (27), Lemma 21] and the fact that $\left|\nabla \eta_{c}\right| \leq 2 / c$, we have

$$
\begin{aligned}
\tilde{I}_{c}\left(u_{c}\right)= & \frac{1}{2} \int_{\mathbb{R}^{3}}\left|\nabla u_{c}\right|^{2}+2 m \mu u_{c}^{2}-q m u_{c}^{2} \phi_{u_{c}} d x-\frac{1}{p} \int_{\mathbb{R}^{3}}\left(u_{c}\right)_{+}^{p} d x \\
& -\frac{1}{2 c^{2}} \int_{\mathbb{R}^{3}} \mu^{2} u_{c}^{2}-q \mu u_{c}^{2} \Phi_{u_{c}} d x-\frac{1}{2} q m \int_{\mathbb{R}^{3}} u_{c}^{2}\left(\Phi_{u_{c}}-\phi_{u_{c}}\right) d x \\
\geq & \tilde{I}_{\infty}\left(v_{c}\right)+\tilde{I}_{\infty}\left(w_{c}\right)+\int_{\mathbb{R}^{3}} \nabla v_{c} \cdot \nabla w_{c}+2 m \mu v_{c} w_{c} d x+o(1) \\
= & \tilde{I}_{\infty}\left(v_{c}\right)+\tilde{I}_{\infty}\left(w_{c}\right)+\int_{\mathbb{R}^{3}}\left(1-\tilde{\eta}_{c}\right) \tilde{\eta}_{c}\left|\nabla u_{c}\right|^{2}+2 m \mu\left(1-\tilde{\eta}_{c}\right) \tilde{\eta}_{c} u_{c}^{2} d x+o(1) \\
\geq & \tilde{I}_{\infty}\left(v_{c}\right)+\tilde{I}_{\infty}\left(w_{c}\right)+o(1)
\end{aligned}
$$

as $c \rightarrow \infty$.

Step 2. $\tilde{\tilde{I}}_{\infty}\left(w_{c}\right) \geq 0$ for large $c$, where $w_{c}=\left(1-\tilde{\eta}_{c}\right) u_{c}$.

We note that, by Lemma 17.

$$
\left|\int_{\mathbb{R}^{3}} w_{c}^{2} \phi_{w_{c}} d x\right| \leq\left\|\phi_{w_{c}}\right\|_{L^{6}}\left\|w_{c}^{2}\right\|_{L^{6 / 5}} \leq C_{2}\left\|w_{c}\right\|_{H^{1}}^{4},
$$

where $C_{2}$ is a positive constant independent of $c$. Moreover, by (21) and Proposition $4 .\left\|w_{c}\right\|_{H^{1}} \leq 2 d$ for large $c>0$. Then we have

$$
\begin{aligned}
\tilde{I}_{\infty}\left(w_{c}\right) & =\frac{1}{2} \int_{\mathbb{R}^{3}}\left|\nabla w_{c}\right|^{2}+2 m \mu w_{c}^{2}-q m w_{c}^{2} \phi_{w_{c}} d x-\frac{1}{p} \int_{\mathbb{R}^{3}}\left(w_{c}\right)_{+}^{p} d x \\
& \geq\left\|w_{c}\right\|_{H^{1}}^{2}\left(\min \left\{\frac{1}{2}, m \mu\right\}-q m C_{2}\left(\left\|w_{c}\right\|_{H^{1}}^{2}+\left\|w_{c}\right\|_{H^{1}}^{p-2}\right)\right) .
\end{aligned}
$$

Taking $d>0$ small, we deduce that $\tilde{I}_{\infty}\left(w_{c}\right) \geq 0$ for large $c$.

Step 3. $v_{c} \rightarrow \tilde{V}(\cdot-z)$ in $H^{1}$, where $\tilde{V} \in \mathcal{A}, z \in \mathbb{R}^{3}$ and $v_{c}=\tilde{\eta}_{c} u_{c}$.

Let $W_{c} \equiv v_{c}\left(\cdot+y_{c}\right)$. We can assume that $W_{c} \rightarrow W \not \equiv 0$ in $H^{1}$, up to a subsequence, as $c \rightarrow \infty$. Since $W_{c}-u_{c}\left(\cdot+y_{c}\right) \rightarrow 0$ in $H^{1}, \phi_{W_{c}}-\phi_{u_{c}\left(\cdot+y_{c}\right)} \rightarrow 0$ in $D^{1,2}$. Then for any $\psi \in C_{0}^{\infty}\left(\mathbb{R}^{3}\right)$,

$$
\begin{gathered}
\int_{\mathbb{R}^{3}}\left(W_{c} \phi_{W_{c}}-u_{c}\left(\cdot+y_{c}\right) \phi_{u_{c}\left(\cdot+y_{c}\right)}\right) \psi d x=\int_{\mathbb{R}^{3}}\left(W_{c}-W\right)\left(\phi_{W_{c}}-\phi_{u_{c}\left(\cdot+y_{c}\right)}\right) \psi+W\left(\phi_{W_{c}}-\phi_{u_{c}\left(\cdot+y_{c}\right)}\right) \psi \\
+\left(W_{c}-u_{c}\left(\cdot+y_{c}\right)\right) \phi_{u_{c}\left(\cdot+y_{c}\right)} \psi d x \rightarrow 0
\end{gathered}
$$

as $c \rightarrow \infty$. From this, (18), (24) and the assumption that $\left\|\tilde{I}_{c}^{\prime}\left(u_{c}\right)\right\|_{H^{-1}} \rightarrow 0$ as $c \rightarrow \infty$, we can see that $W$ satisfies $\tilde{I}_{\infty}^{\prime}(W)=0$. By the maximum principle, $W$ is positive. Suppose that there exist $R>0$ and a sequence $\tilde{z}_{c} \in B\left(y_{c}, 2 c\right)$ satisfying

$$
\liminf _{c \rightarrow \infty}\left|\tilde{z}_{c}-y_{c}\right|=\infty \text { and } \liminf _{c \rightarrow \infty} \int_{B\left(\tilde{z}_{c}, R\right)}\left|v_{c}\right|^{2} d x>0 .
$$

Then $v_{c}\left(\cdot+z_{c}\right)$ converges weakly to $\tilde{W}$ in $H^{1}$, where $I_{\infty}^{\prime}(\tilde{W})=0$. By the same arguments in Step 1 , we deduce a contradiction. By [13, Lemma 1.1], we have

$$
\lim _{c \rightarrow \infty} \int_{\mathbb{R}^{3}}\left(W_{c}\right)_{+}^{p} d x=\int_{\mathbb{R}^{3}} W^{p} d x
$$

We note that

$$
\begin{aligned}
\liminf _{c \rightarrow \infty}\left(-\int_{\mathbb{R}^{3}} W_{c}^{2} \phi_{W_{c}} d x\right) & =\liminf _{c \rightarrow \infty} \int_{\mathbb{R}^{3}} \int_{\mathbb{R}^{3}} \frac{W_{c}^{2}(x) W_{c}^{2}(y)}{|x-y|} d y d x \\
& \geq \int_{\mathbb{R}^{3}} \int_{\mathbb{R}^{3}} \frac{W^{2}(x) W^{2}(y)}{|x-y|} d y d x=-\int_{\mathbb{R}^{3}} W^{2} \phi_{W} d x .
\end{aligned}
$$

Then, by (29), (30) and Lemma 21, we have

$$
\begin{aligned}
\liminf _{c \rightarrow \infty} \tilde{I}_{\infty}\left(W_{c}\right) & =\liminf _{c \rightarrow \infty} \frac{1}{2} \int_{\mathbb{R}^{3}}\left|\nabla W_{c}\right|^{2}+2 m \mu W_{c}^{2}-q m W_{c}^{2} \phi_{W_{c}} d x-\frac{1}{p} \int_{\mathbb{R}^{3}}\left(W_{c}\right)_{+}^{p} d x \\
& \geq \tilde{I}_{\infty}(W) .
\end{aligned}
$$


By (31), the results of Step1 and Step 2, and the assumption that $\tilde{I}_{c}\left(u_{c}\right) \leq \hat{e}_{c}$, we see that $\tilde{I}_{\infty}(W)=$ $E_{\infty}$. By (29), (30) and (31), we have

$$
\begin{aligned}
\limsup _{c \rightarrow \infty} & \int_{\mathbb{R}^{3}}\left|\nabla W_{c}\right|^{2}+2 m \mu W_{c}^{2}-q m W_{c}^{2} \phi_{W_{c}} d x=\int_{\mathbb{R}^{3}}|\nabla W|^{2}+2 m \mu W^{2}-q m W^{2} \phi_{W} d x \\
& \leq \int_{\mathbb{R}^{3}}|\nabla W|^{2}+2 m \mu W^{2}+\limsup _{c \rightarrow \infty}\left(-\int_{\mathbb{R}^{3}} q m W_{c}^{2} \phi_{W_{c}} d x\right),
\end{aligned}
$$

which implies that $W_{c} \rightarrow W$ in $H^{1}$. By (28), the result of Step 1 and the fact that $\hat{e}_{c} \rightarrow E_{\infty}$, we have for small $d>0$,

$$
\hat{e}_{c} \geq \tilde{I}_{c}\left(u_{c}\right) \geq \tilde{I}_{\infty}\left(v_{c}\right)+\frac{1}{2} \min \left\{\frac{1}{2}, m \mu\right\}\left\|w_{c}\right\|_{H^{1}}^{2}+o(1) \geq E_{\infty}+\frac{1}{2} \min \left\{\frac{1}{2}, m \mu\right\}\left\|w_{c}\right\|_{H^{1}}^{2}+o(1)
$$

as $c \rightarrow \infty$, which implies that $\left\|w_{c}\right\|_{H^{1}} \rightarrow 0$ as $c \rightarrow \infty$. Thus, letting $W=\tilde{V}(\cdot-z)$, where $\tilde{V} \in \mathcal{A}$ and $z \in \mathbb{R}^{3}$, we have

$$
\left\|u_{c}-\tilde{V}\left(\cdot-y_{c}-z\right)\right\|_{H^{1}} \leq\left\|v_{c}\left(\cdot+y_{c}\right)-\tilde{V}(\cdot-z)\right\|_{H^{1}}+\left\|w_{c}\right\|_{H^{1}} \rightarrow 0
$$

as $c \rightarrow \infty$.

Proposition 8. Let $3<p<6$. For a fixed $c \in\left(\sqrt{\frac{\mu}{m}}, \infty\right)$, suppose that for some $b \in \mathbb{R}$, there exists a sequence $\left\{u_{j}\right\} \subset H^{1}$ satisfying

$$
\begin{aligned}
& u_{j} \in N_{d}(\mathcal{X}), \\
& \left\|\tilde{I}_{c}^{\prime}\left(u_{j}\right)\right\|_{H^{-1}} \rightarrow 0, \\
& \tilde{I}_{c}\left(u_{j}\right) \rightarrow b \text { as } j \rightarrow \infty,
\end{aligned}
$$

where $d>0$ is a constant. Then for small $d>0, b$ is a critical value of $\tilde{I}_{c}$, and the sequence $\left\{u_{j}\left(\cdot+x_{j}\right)\right\}_{j=1}^{\infty} \subset H^{1}$ has a strongly convergent subsequence in $H^{1}$, where $x_{j} \in \mathbb{R}^{3}$.

Proof. Since $u_{j} \in N_{d}(\mathcal{X}),\left\{u_{j}\right\}_{j=1}^{\infty}$ is bounded in $H^{1}$. Then we can extract a subsequence such that $\tilde{u}_{j_{k}} \equiv u_{j_{k}}\left(\cdot+x_{j_{k}}\right)$ converges to $u_{0} \not \equiv 0$ weakly in $H^{1}$ as $k \rightarrow \infty$, where $x_{j_{k}} \in \mathbb{R}^{3}$. It is standard to show that $u_{0}$ is a critical point of $I_{c}$.

Next, we show $\tilde{u}_{j_{k}} \rightarrow u_{0}$ in $H^{1}$ as $k \rightarrow \infty$. By Proposition 4 there exists $R_{0}>0$ such that

$$
\left\|\tilde{u}_{j_{k}}\right\|_{H^{1}\left(\mathbb{R}^{3} \backslash B\left(0, R_{0}\right)\right)} \leq 2 d .
$$

We choose a function $\zeta \in C^{\infty}\left(\mathbb{R}^{3}\right)$ such that

$$
\zeta(x)= \begin{cases}1 & \text { for }|x| \geq 2 R_{0}, \\ 0 & \text { for }|x| \leq R_{0} .\end{cases}
$$

Since $\tilde{I}_{c}^{\prime}\left(\tilde{u}_{j_{k}}\right)\left(\zeta\left(\tilde{u}_{j_{k}}-u_{0}\right)\right)-\tilde{I}_{c}^{\prime}\left(u_{0}\right)\left(\zeta\left(\tilde{u}_{j_{k}}-u_{0}\right)\right) \rightarrow 0$ as $k \rightarrow \infty$, we deduce that

$$
\begin{aligned}
& \int_{\mathbb{R}^{3} \backslash B(0,2 R)}\left|\nabla\left(\tilde{u}_{j_{k}}-u_{0}\right)\right|^{2}+\left(2 m \mu-\frac{\mu^{2}}{c^{2}}\right)\left(\tilde{u}_{j_{k}}-u_{0}\right)^{2} d x \\
& \leq \int_{\mathbb{R}^{3} \backslash B(0,2 R)}\left(\frac{q}{c}\right)^{2}\left(\tilde{u}_{j_{k}}-u_{0}\right)\left(\tilde{u}_{j_{k}} \Phi_{\tilde{u}_{j_{k}}}^{2}-u_{0} \Phi_{u_{0}}^{2}\right)+2 q\left(m-\frac{\mu}{c^{2}}\right)\left(\tilde{u}_{j_{k}}-u_{0}\right)\left(\tilde{u}_{j_{k}} \Phi_{\tilde{u}_{j_{k}}}-u_{0} \Phi_{u_{0}}\right) \\
& \quad+\left(\tilde{u}_{j_{k}}-u_{0}\right)\left(\left(\tilde{u}_{j_{k}}\right)_{+}^{p-1}-\left(u_{0}\right)_{+}^{p-1}\right) d x+o(1)
\end{aligned}
$$

as $k \rightarrow \infty$. We note that, by Lemma 18 ,

$$
\begin{aligned}
& \int_{\mathbb{R}^{3} \backslash B(0,2 R)}(v-w)\left(v \Phi_{v}^{2}-w \Phi_{w}^{2}\right) d x \\
& \leq\left(\left\|\Phi_{v}\right\|_{L^{6}}^{2}\|v\|_{L^{3}\left(\mathbb{R}^{3} \backslash B(0,2 R)\right)}+\left\|\Phi_{w}\right\|_{L^{6}}^{2}\|w\|_{L^{3}\left(\mathbb{R}^{3} \backslash B(0,2 R)\right)}\right)\|v-w\|_{L^{3}\left(\mathbb{R}^{3} \backslash B(0,2 R)\right)} \\
& \leq C_{1}\left(\|v\|_{H^{1}}^{4}\|v\|_{H^{1}\left(\mathbb{R}^{3} \backslash B(0,2 R)\right)}+\|w\|_{H^{1}}^{4}\|w\|_{H^{1}\left(\mathbb{R}^{3} \backslash B(0,2 R)\right)}\right)\|v-w\|_{H^{1}\left(\mathbb{R}^{3} \backslash B(0,2 R)\right)},
\end{aligned}
$$




$$
\begin{aligned}
& \int_{\mathbb{R}^{3} \backslash B(0,2 R)}(v-w)\left(v \Phi_{v}-w \Phi_{w}\right) d x \\
& \leq\left(\left\|\Phi_{v}\right\|_{L^{6}}\|v\|_{L^{3}\left(\mathbb{R}^{3} \backslash B(0,2 R)\right)}+\left\|\Phi_{w}\right\|_{L^{6}}\|w\|_{L^{3}\left(\mathbb{R}^{3} \backslash B(0,2 R)\right)}\right)\|v-w\|_{L^{2}\left(\mathbb{R}^{3} \backslash B(0,2 R)\right)} \\
& \leq C_{2}\left(\|v\|_{H^{1}}^{2}\|v\|_{H^{1}\left(\mathbb{R}^{3} \backslash B(0,2 R)\right)}+\|w\|_{H^{1}}^{2}\|w\|_{H^{1}\left(\mathbb{R}^{3} \backslash B(0,2 R)\right)}\right)\|v-w\|_{H^{1}\left(\mathbb{R}^{3} \backslash B(0,2 R)\right)},
\end{aligned}
$$

and

$$
\begin{aligned}
& \int_{\mathbb{R}^{3} \backslash B(0,2 R)}\left((v)_{+}^{p-1}-(w)_{+}^{p-1}\right)(v-w) d x=(p-1) \int_{\mathbb{R}^{3} \backslash B(0,2 R)}(t v+(1-t) w)_{+}^{p-2}(v-w)^{2} d x \\
& \leq(p-1)\|t v+(1-t) w\|_{L^{p}\left(\mathbb{R}^{3} \backslash B(0,2 R)\right)}^{p-2}\|v-w\|_{L^{p}\left(\mathbb{R}^{3} \backslash B(0,2 R)\right)}^{2} \\
& \leq C_{3}\left(\|v\|_{H^{1}\left(\mathbb{R}^{3} \backslash B(0,2 R)\right)}^{p-2}+\|w\|_{H^{1}\left(\mathbb{R}^{3} \backslash B(0,2 R)\right)}^{p-2}\right)\|v-w\|_{H^{1}\left(\mathbb{R}^{3} \backslash B(0,2 R)\right)}^{2},
\end{aligned}
$$

where $t \in[0,1]$. Then, by (32)-(36), we see that for small $d>0$,

$$
\left\|\tilde{u}_{j_{k}}-u_{0}\right\|_{H^{1}\left(\mathbb{R}^{3} \backslash B(0,2 R)\right)} \rightarrow 0
$$

as $k \rightarrow \infty$. Thus, by (37) and the Rellich-Kondrachov compactness theorem, we see that $\tilde{u}_{j_{k}} \rightarrow u_{0}$ in $H^{1}$ as $k \rightarrow \infty$.

Proposition 9. For $3<p<6$, there exist $\bar{c}_{0}>0$ and $\bar{d}_{0}>0$ such that for $c>\bar{c}_{0}$ and for $0<d<\bar{d}_{0}, \tilde{I}_{c}$ has a critical point $u$ in $N_{d}(\mathcal{X})$ with $\tilde{I}_{c}(u) \leq \hat{e}_{c}$.

Proof. Arguing indirectly, suppose $\tilde{I}_{c}^{\prime}(u) \neq 0$ for $u \in N_{d}(\mathcal{X})$ with $\tilde{I}_{c}(u) \leq \hat{e}_{c}$. By Proposition 7 and Proposition 8 we can take positive constants $\bar{c}_{0}$ and $\bar{d}_{0}$ such that for $c>\bar{c}_{0}$ and for $0<d<\bar{d}_{0}$,

$$
\left\|\tilde{I}_{c}^{\prime}(u)\right\|_{H^{-1}} \geq \nu
$$

for $u \in N_{d}(\mathcal{X}) \backslash N_{d / 2}(\mathcal{X})$ with $\tilde{I}_{c}(u) \leq \hat{e}_{c}$, and

$$
\left\|\tilde{I}_{c}^{\prime}(u)\right\|_{H^{-1}} \geq \sigma_{c}
$$

for $u \in N_{d}(\mathcal{X})$ with $\tilde{I}_{c}(u) \leq \hat{e}_{c}$, where $\nu>0$ is a constant independent of $c$, and $\sigma_{c}>0$ is a constant depending on $c$. Then, by a deformation argument using Proposition 5 and Proposition 6 (see Proposition 7 in [5] for a detailed argument), we get a contradiction.

\section{Nonrelativistic limit of Ground States for $3<p<6$}

In this section, we complete the proof of Theorem [1. By Proposition [3. Proposition [5 and Proposition 9, we see that for every $3<p<6$, there exists a ground state solutions $u_{c}$ to (5) such that

$$
\limsup _{c \rightarrow \infty} I_{c}\left(u_{c}\right) \leq E_{\infty} .
$$

Proposition 10. Let $3<p<6$ and $u_{c}$ be a ground state solution of (5). Then we have

$$
\sup _{c>\sqrt{\frac{\mu}{m}}}\left\|u_{c}\right\|_{H^{1}} \leq C \text { and } \inf _{c>\sqrt{\frac{\mu}{m}}}\left\|u_{c}\right\|_{L^{p}} \geq \frac{1}{C},
$$

where $C>0$ is a constant independent of $c$.

Proof. We note by (38) that

$$
\begin{aligned}
C_{1} & \geq \frac{5 p-12}{2} I_{c}\left(u_{c}\right)-J_{c}\left(u_{c}\right)+\frac{4-p}{2} P_{c}\left(u_{c}\right) \\
& =\int_{\mathbb{R}^{3}}(p-3)\left|\nabla u_{c}\right|^{2}+\frac{p-2}{2}\left(2 m \mu-\frac{\mu^{2}}{c^{2}}\right) u_{c}^{2}+\frac{p-2}{2}\left(\frac{q}{c}\right)^{2} u_{c}^{2} \Phi_{u_{c}}^{2} d x,
\end{aligned}
$$


where $C_{1}>0$ is a constant independent of $c$. This implies $\left\|u_{c}\right\|_{H^{1}}$ is bounded uniformly in $c>\sqrt{\frac{\mu}{m}}$. Moreover, since $J_{c}\left(u_{c}\right)=0$ and $-\frac{1}{q}\left(c^{2} m-\mu\right) \leq \Phi_{u_{c}} \leq 0$, we have for $c>\sqrt{\frac{\mu}{m}}$,

$$
\begin{aligned}
\int_{\mathbb{R}^{3}}\left|u_{c}\right|^{p} & =\int_{\mathbb{R}^{3}}\left|\nabla u_{c}\right|^{2}+\left(2 m \mu-\frac{\mu^{2}}{c^{2}}\right) u_{c}^{2}-\left(\frac{q}{c}\right)^{2} \Phi_{u_{c}} u_{c}^{2}\left(\Phi_{u_{c}}+\left(\frac{c}{q}\right)^{2} 2 q\left(m-\frac{\mu}{c^{2}}\right)\right) d x \\
& \geq \int_{\mathbb{R}^{3}}\left|\nabla u_{c}\right|^{2}+m \mu u_{c}^{2} d x+\left(\frac{q}{c}\right)^{2}\left|\Phi_{u_{c}}\right| u_{c}^{2}\left(\Phi_{u_{c}}+2 \frac{1}{q}\left(c^{2} m-\mu\right)\right) d x \\
& \geq \int_{\mathbb{R}^{3}}\left|\nabla u_{c}\right|^{2}+m \mu u_{c}^{2} d x \geq C_{2}\left(\int_{\mathbb{R}^{3}}\left|u_{c}\right|^{p} d x\right)^{2 / p}
\end{aligned}
$$

where $C_{2}$ is a positive constant indendent of $c$. Then we have $\int_{\mathbb{R}^{3}}\left|u_{c}\right|^{p} d x \geq \frac{1}{C}$, where $C$ is a positive constant indendent of $c$.

Proposition 11. For $3<p<6$, let $\left\{u_{c}\right\}_{c>\sqrt{\frac{\mu}{m}}} \subset H^{1}$ be a ground state solution of (55). Then there exists a sequence $\left\{x_{c}\right\} \in \mathbb{R}^{3}$ such that $\bar{u}_{c}(\cdot) \equiv u_{c}\left(\cdot+x_{c}\right)$ converges to $u_{\infty}$ in $H^{1}\left(\mathbb{R}^{3}\right)$ as $c \rightarrow \infty$, up to a subsequence, where $u_{\infty}$ is a ground state solution of (10).

Proof. By Proposition 10 and [13, Lemma 1.1], we have

$$
\sup _{x \in \mathbb{R}^{3}} \int_{B_{1}(x)}\left|u_{c}\right|^{2} d x=\int_{B_{1}\left(x_{c}\right)}\left|u_{c}\right|^{2} d x \geq \bar{C}>0,
$$

where $\bar{C}$ is a constant indepnedent of $c$.

It follows from Proposition [10 that $\left\{u_{c}\right\}_{c>\sqrt{\frac{\mu}{m}}}$ is bounded in $H^{1}$ uniformly in $c$. Then we may assume $\bar{u}_{c} \equiv u_{c}\left(\cdot+x_{c}\right)$ converges to $u_{\infty} \not \equiv 0$ weakly in $H^{1}$ and strongly in $L_{l o c}^{q}\left(\mathbb{R}^{3}\right)$, where $0<q<6$. Let $\Phi_{\bar{u}_{c}}$ be the solution of

$$
-\Delta \Phi+\frac{q^{2}}{c^{2}} \bar{u}_{c}^{2} \Phi=-q\left(m-\frac{\mu}{c^{2}}\right) \bar{u}_{c}^{2} \text { in } \mathbb{R}^{3} .
$$

Since $\left\|\Phi_{\bar{u}_{c}}\right\|_{D^{1,2}} \leq C_{1} q\left(m-\frac{\mu}{c^{2}}\right)\left\|\bar{u}_{c}\right\|_{H^{1}}^{2} \leq C_{2}$, where $C_{1}, C_{2}>0$ are constants independent of $c$, we may assume that

$$
\Phi_{\bar{u}_{c}} \rightarrow \phi_{u_{\infty}} \text { weakly in } D^{1,2} \text { and } \Phi_{\bar{u}_{c}} \rightarrow \phi_{u_{\infty}} \text { in } L_{l o c}^{q}\left(\mathbb{R}^{3}\right),
$$

as $c \rightarrow \infty$, where $0<q<6$ and $\phi_{u_{\infty}}$ is a weak solution of $-\Delta \phi+q m u_{\infty}^{2}=0$. Then it is standard to show that $u_{\infty}$ is a non-trivial weak solution of (10).

Next, we claim that $u_{\infty}$ is a ground state solution of (10). We note that, since $u_{\infty}$ is a non-trivial weak solution of (10), we have

$$
J_{\infty}\left(u_{\infty}\right)=P_{\infty}\left(u_{\infty}\right)=0
$$

and

$$
\frac{5 p-12}{2} I_{\infty}\left(u_{\infty}\right)-J_{\infty}\left(u_{\infty}\right)+\frac{4-p}{2} P_{\infty}\left(u_{\infty}\right)=\int_{\mathbb{R}^{3}}(p-3)\left|\nabla u_{\infty}\right|^{2}+(p-2) m \mu u_{\infty}^{2} .
$$

Then, by (38), (39) and (41), we have

$$
\begin{aligned}
\frac{5 p-12}{2} E_{\infty} & \geq \frac{5 p-12}{2} \liminf _{c \rightarrow \infty} I_{c}\left(u_{c}\right) \\
& =\liminf _{c \rightarrow \infty}\left(\int_{\mathbb{R}^{3}}(p-3)\left|\nabla u_{c}\right|^{2}+\frac{p-2}{2}\left(2 m \mu-\frac{\mu^{2}}{c^{2}}\right) u_{c}^{2}+\frac{p-2}{2}\left(\frac{q}{c}\right)^{2} u_{c}^{2} \Phi_{u_{c}}^{2} d x\right) \\
& \geq \int_{\mathbb{R}^{3}}(p-3)\left|\nabla u_{\infty}\right|^{2}+(p-2) m \mu u_{\infty}^{2}=\frac{5 p-12}{2} I_{\infty}\left(u_{\infty}\right),
\end{aligned}
$$

which proves the claim. 
Finally, to prove the strong convergence in $H^{1}\left(\mathbb{R}^{3}\right)$, we note that, by (38), (39), (41), Proposition 10 and the fact that $\bar{u}_{c}$ converges to $u_{\infty} \not \equiv 0$ weakly in $H^{1}$,

$$
\begin{aligned}
\frac{5 p-12}{2} E_{\infty} \geq & \frac{5 p-12}{2} \lim _{c \rightarrow \infty} I_{c}\left(\bar{u}_{c}\right) \\
= & \lim _{c \rightarrow \infty} \int_{\mathbb{R}^{3}}(p-3)\left|\nabla \bar{u}_{c}\right|^{2}+\frac{p-2}{2}\left(2 m \mu-\frac{\mu^{2}}{c^{2}}\right) \bar{u}_{c}^{2}+\frac{p-2}{2}\left(\frac{q}{c}\right)^{2} \bar{u}_{c}^{2} \Phi_{\bar{u}_{c}}^{2} d x \\
= & \int_{\mathbb{R}^{3}}(p-3)\left|\nabla u_{\infty}\right|^{2}+(p-2) m \mu u_{\infty}^{2} d x \\
& \quad+\lim _{c \rightarrow \infty} \int_{\mathbb{R}^{3}}(p-3)\left|\nabla\left(\bar{u}_{c}-u_{\infty}\right)\right|^{2}+(p-2) m \mu\left(\bar{u}_{c}-u_{\infty}\right)^{2} d x \\
= & \frac{5 p-12}{2} E_{\infty}+\lim _{c \rightarrow \infty} \int_{\mathbb{R}^{3}}(p-3)\left|\nabla\left(\bar{u}_{c}-u_{\infty}\right)\right|^{2}+(p-2) m \mu\left(\bar{u}_{c}-u_{\infty}\right)^{2} d x .
\end{aligned}
$$

From this, we deduce that $\bar{u}_{c} \rightarrow u_{\infty}$ in $H^{1}$ as $c \rightarrow \infty$, up to a subsequence. This completes the proof.

Proof of Theorem 1. It is sufficient to show $H^{2}$ convergence of $\bar{u}_{c}$ to $u_{\infty}$. We may rewrite $\bar{u}_{c}$ as $u_{c}$. We note that, by Lemma 18 and [20, Theorem 4.1], for $u \in H^{1}$,

$$
\sup _{x \in \Omega}\left|\Phi_{u}(x)\right| \leq C_{1}\|u\|_{H^{1}}^{2} \quad \text { and } \quad\left\||u|^{p-2}\right\|_{L^{\frac{6}{p-2}}(\Omega)}=\|u\|_{L^{6}(\Omega)}^{p-2} \leq C_{2}\|u\|_{H^{1}}^{p-2},
$$

where $\Omega$ is bounded domain in $\mathbb{R}^{3}$, and $C_{1}$ and $C_{2}$ are positive constants independent of $u$ and $\Omega$. Then, since $\left\{\left\|u_{c}\right\|_{H^{1}}\right\}_{c}$ is bounded, we see that $\left\{\left\|u_{c}\right\|_{L^{\infty}}\right\}_{c}$ is bounded (see [20, Theorem 4.1]).

Since $u_{\infty}$ and $u_{c}$ are solutions of (10) and (5) respectively, we have

$$
\begin{aligned}
-\Delta\left(u_{c}-u_{\infty}\right)=- & 2 m \mu\left(u_{c}-u_{\infty}\right)+\left(\frac{\mu}{c}\right)^{2} u_{c}+\left(\frac{q}{c}\right)^{2} u_{c} \Phi_{u_{c}}^{2}-2 q \frac{\mu}{c^{2}} u_{c} \Phi_{u_{c}} \\
& +2 q m\left(u_{c} \Phi_{u_{c}}-u_{\infty} \phi_{u_{\infty}}\right)+\left|u_{c}\right|^{p-2} u_{c}-\left|u_{\infty}\right|^{p-2} u_{\infty} .
\end{aligned}
$$

We note that, by Lemma 17. Lemma 19, Lemma 21 and Proposition 11

$$
\begin{aligned}
& \left\|u_{c} \Phi_{u_{c}}-u_{\infty} \phi_{u_{\infty}}\right\|_{L^{2}} \\
& =\left\|u_{c}\left(\Phi_{u_{c}}-\phi_{u_{c}}\right)+\left(u_{c}-u_{\infty}\right) \phi_{u_{c}}+\left(\phi_{u_{c}}-\phi_{u_{\infty}}\right) u_{\infty}\right\|_{L^{2}} \\
& \leq\left\|u_{c}\right\|_{L^{3}}\left\|\Phi_{u_{c}}-\phi_{u_{c}}\right\|_{L^{6}}+\left\|u_{c}-u_{\infty}\right\|_{L^{3}}\left\|\phi_{u_{c}}\right\|_{L^{6}}+\left\|\phi_{u_{c}}-\phi_{u_{\infty}}\right\|_{L^{6}}\left\|u_{\infty}\right\|_{L^{3}} \rightarrow 0
\end{aligned}
$$

as $c \rightarrow \infty$, and by the fact that $\left\{\left\|u_{c}\right\|_{L^{\infty}}\right\}_{c}$ is bounded,

$$
\left\|\left|u_{c}\right|^{p-2} u_{c}-\left|u_{\infty}\right|^{p-2} u_{\infty}\right\|_{L^{2}}=(p-1)\left\|\left|u_{\infty}+t\left(u_{c}-u_{\infty}\right)\right|^{p-2}\left(u_{c}-u_{\infty}\right)\right\|_{L^{2}} \rightarrow 0
$$

as $c \rightarrow \infty$, where $t \in[0,1]$. Thus, by (42)-(44) and the Calderón-Zygmund inequality, we have

$$
\left\|u_{c}-u_{\infty}\right\|_{H^{2}\left(\mathbb{R}^{3}\right)}=\left\|-\Delta\left(u_{c}-u_{\infty}\right)\right\|_{L^{2}}+o(1)=o(1)
$$

as $c \rightarrow \infty$.

\section{Nonrelativistic limit of two POSitive solutions fOR $2<p<3$}

In this section, we will construct two radially symmetric positive solutions of NMKG for $2<p<3$. We prove first the existence of a radially symmetric positive solution $v_{c, q}$ of (5) satisfying

$$
\lim _{c \rightarrow \infty}\left\|v_{c, q}-v_{\infty}\right\|_{H^{1}}=0,
$$

where $v_{\infty}$ is a global minimizer of $I_{\infty}$.

We assume $2<p<3$ and denote

$$
e_{\infty} \equiv \inf _{u \in H_{r}^{1}} \tilde{I}_{\infty}(u), \quad \mathcal{X}_{r} \equiv\left\{u \in H_{r}^{1} \mid \tilde{I}_{\infty}(u)=e_{\infty}\right\}
$$

and

$$
N_{d}\left(\mathcal{X}_{r}\right) \equiv\left\{u \in H_{r}^{1} \mid \inf _{v \in \mathcal{X}_{r}}\|u-v\|_{H^{1}} \leq d\right\}
$$


where $d>0$ is a constant. We remark that, by [19. Theorem 4.3, Corollary 4.4], $\mathcal{X}_{r}$ is bounded in $H^{1}$, and for small $q>0, e_{\infty}<0$ and $\mathcal{X}_{r} \neq \emptyset$. Moreover, since $e_{\infty}<0$ for small $q>0$, and for $u \in \mathcal{X}_{r}$

$$
\begin{aligned}
e_{\infty}=\tilde{I}_{\infty}(u) & =\frac{1}{2} \int_{\mathbb{R}^{3}}|\nabla u|^{2}+2 m \mu u^{2}-q m u^{2} \phi_{u} d x-\frac{1}{p} \int_{\mathbb{R}^{3}}(u)_{+}^{p} d x \\
& \geq \frac{1}{2} \int_{\mathbb{R}^{3}}|\nabla u|^{2}+2 m \mu u^{2} d x-\frac{C_{1}}{p}\left(\int_{\mathbb{R}^{3}}|\nabla u|^{2}+u^{2} d x\right)^{p / 2},
\end{aligned}
$$

where $C_{1}>0$ is a constant independent of $u \in \mathcal{X}_{r}$, we see that there exists $\hat{q}_{0}>0$ such that for $0<q<\hat{q}_{0}, \mathcal{X}_{r} \neq \emptyset$ and

$$
\inf _{u \in \mathcal{X}_{r}}\|u\|_{H^{1}}>\hat{d}_{0}>0
$$

where $\hat{d}_{0}$ is a positive constant. Taking $d \in\left(0, \frac{\hat{d}_{0}}{2}\right)$, we deduce that for $0<q<\hat{q}_{0}, 0 \notin N_{d}\left(\mathcal{X}_{r}\right)$. For $d \in\left(0, \frac{\hat{d}_{0}}{2}\right)$ and $0<q<\hat{q}_{0}$, take $V_{0} \in \mathcal{X}_{r}$ and set

$$
\alpha_{c}=\inf _{u \in N_{d}\left(\mathcal{X}_{r}\right)} \tilde{I}_{c}(u) \text { and } m_{c}=\tilde{I}_{c}\left(V_{0}\right) .
$$

Clearly, we have $m_{c} \geq \alpha_{c}$. We try to find a critical point of $\tilde{I}_{c}$ in $N_{d}\left(\mathcal{X}_{r}\right)$.

Proposition 12. For $2<p<3,0<q<\hat{q}_{0}$ and $d \in\left(0, \frac{\hat{d}_{0}}{2}\right)$, we have

$$
\liminf _{c \rightarrow \infty} \alpha_{c} \geq e_{\infty} .
$$

Proof. It is standard to show that there exists $v_{c} \in N_{d}\left(\mathcal{X}_{r}\right)$ such that

$$
\alpha_{c}=\tilde{I}_{c}\left(v_{c}\right) \text {, }
$$

because $\mathcal{X}_{r}$ is bounded in $H^{1}$. Since $v_{c}$ is bounded in $H_{r}^{1}$ uniformly in $c$, we assume that $v_{c}$ converges to $v$ in $L^{s}$ and weakly in $H^{1}$ as $c \rightarrow \infty$, where $s \in(2,6)$ and $v \in N_{d}\left(\mathcal{X}_{r}\right)$. Then, by Lemma 21, we have

$$
\begin{aligned}
\liminf _{c \rightarrow \infty} \alpha_{c} & =\liminf _{c \rightarrow \infty} \tilde{I}_{c}\left(v_{c}\right) \\
& =\liminf _{c \rightarrow \infty}\left[\frac{1}{2} \int_{\mathbb{R}^{3}}\left|\nabla v_{c}\right|^{2}+\left(2 m \mu-\frac{\mu^{2}}{c^{2}}\right) v_{c}^{2}-q\left(m-\frac{\mu}{c^{2}}\right) v_{c}^{2} \Phi_{v_{c}} d x-\frac{1}{p} \int_{\mathbb{R}^{3}}\left(v_{c}\right)_{+}^{p} d x\right] \\
& \geq \frac{1}{2} \int_{\mathbb{R}^{3}}|\nabla v|^{2}+2 m \mu v^{2}-q m v^{2} \phi_{v} d x-\frac{1}{p} \int_{\mathbb{R}^{3}}(v)_{+}^{p} d x=\tilde{I}_{\infty}(v) \geq e_{\infty} .
\end{aligned}
$$

Proposition 13. For $2<p<3$ and $0<q<\hat{q}_{0}$, we have

$$
m_{c} \rightarrow e_{\infty}
$$

uniformly in $q$ as $c \rightarrow \infty$.

Proof. By Lemma 21.

$$
\begin{aligned}
\tilde{I}_{c}\left(V_{0}\right) & =\frac{1}{2} \int_{\mathbb{R}^{3}}\left|\nabla V_{0}\right|^{2}+\left(2 m \mu-\frac{\mu^{2}}{c^{2}}\right) V_{0}^{2}-q\left(m-\frac{\mu}{c^{2}}\right) V_{0}^{2} \Phi_{V_{0}} d x-\frac{1}{p} \int_{\mathbb{R}^{3}}\left(V_{0}\right)_{+}^{p} d x \\
& =\frac{1}{2} \int_{\mathbb{R}^{3}}\left|\nabla V_{0}\right|^{2}+2 m \mu V_{0}^{2}-q m V_{0}^{2} \phi_{V_{0}} d x-\frac{1}{p} \int_{\mathbb{R}^{3}}\left(V_{0}\right)_{+}^{p} d x+o(1) \\
& =\tilde{I}_{\infty}\left(V_{0}\right)+o(1)=e_{\infty}+o(1)
\end{aligned}
$$

as $c \rightarrow \infty$.

Proposition 14. Let $2<p<3,0<q<\hat{q}_{0}$ and $d \in\left(0, \frac{\hat{d}_{0}}{2}\right)$. For large $c>0$ and for any $d^{\prime} \in(0, d)$, there exists $\nu_{0} \equiv \nu_{0}\left(d, d^{\prime}\right)>0$ independent of $c>0$ such that

$$
\inf \left\{\left\|\tilde{I}_{c}^{\prime}(u)\right\|_{H^{-1}} \mid \tilde{I}_{c}(u) \leq m_{c}, u \in N_{d}\left(\mathcal{X}_{r}\right) \backslash N_{d^{\prime}}\left(\mathcal{X}_{r}\right)\right\} \geq \nu_{0}>0 .
$$


Proof. Let $\left\{c_{i}\right\}_{i=1}^{\infty}$ be such that $\lim _{i \rightarrow \infty} c_{i}=\infty$. It suffices to show that if

$$
u_{c_{i}} \in N_{d}\left(\mathcal{X}_{r}\right), \quad \tilde{I}_{c_{i}}\left(u_{c_{i}}\right) \leq m_{c_{i}}, \quad \text { and }\left\|\tilde{I}_{c_{i}}^{\prime}\left(u_{c_{i}}\right)\right\|_{H^{-1}} \rightarrow 0
$$

as $i \rightarrow \infty$, then

$$
\inf _{v \in \mathcal{X}_{r}}\left\|u_{c_{i}}-v\right\|_{H^{1}} \rightarrow 0 \text { as } i \rightarrow \infty
$$

For the sake of simplicity of notation, we write $c$ for $c_{i}$. Since $\left\{u_{c}\right\} \subset H_{r}^{1}$ is bounded in $H^{1}$, we see that $u_{c}$ converges to $u$ in $L^{s}$ and weakly in $H^{1}$ as $c \rightarrow \infty$, up to a subsequence, where $s \in(2,6)$. Then, by Lemma 21 and Proposition 13, we have

$$
\begin{aligned}
e_{\infty} & =\liminf _{c \rightarrow \infty} m_{c} \geq \liminf _{c \rightarrow \infty} \tilde{I}_{c}\left(u_{c}\right) \\
& =\liminf _{c \rightarrow \infty}\left[\frac{1}{2} \int_{\mathbb{R}^{3}}\left|\nabla u_{c}\right|^{2}+\left(2 m \mu-\frac{\mu^{2}}{c^{2}}\right) u_{c}^{2}-q\left(m-\frac{\mu}{c^{2}}\right) u_{c}^{2} \Phi_{u_{c}} d x-\frac{1}{p} \int_{\mathbb{R}^{3}}\left(u_{c}\right)_{+}^{p} d x\right] \\
& \geq \frac{1}{2} \int_{\mathbb{R}^{3}}|\nabla u|^{2}+2 m \mu u^{2}-q m u^{2} \phi_{u} d x-\frac{1}{p} \int_{\mathbb{R}^{3}}(u)_{+}^{p} d x=\tilde{I}_{\infty}(u),
\end{aligned}
$$

which implies that $e_{\infty}=\tilde{I}_{\infty}(u)$.

We claim that $u_{c} \rightarrow u$ in $H^{1}$. Indeed, by Lemma21 and the fact that $\left\|\tilde{I}_{c}^{\prime}\left(u_{c}\right)\right\|_{H^{-1}} \rightarrow 0$ as $c \rightarrow \infty$, we see that

$$
\begin{aligned}
o(1) & =\tilde{I}_{c}^{\prime}\left(u_{c}\right) u \\
& =\int_{\mathbb{R}^{3}} \nabla u_{c} \cdot \nabla u+\left(2 m \mu-\frac{\mu^{2}}{c^{2}}\right) u_{c} u-\left(\frac{q}{c}\right)^{2} u_{c} u \Phi_{u_{c}}^{2}-2 q\left(m-\frac{\mu}{c^{2}}\right) u_{c} u \Phi_{u_{c}}-\left(u_{c}\right)_{+}^{p-1} u d x \\
& =\int_{\mathbb{R}^{3}}|\nabla u|^{2}+2 m \mu u^{2}-2 q m u^{2} \phi_{u}-(u)_{+}^{p} d x+o(1)
\end{aligned}
$$

as $c \rightarrow \infty$, and

$$
\begin{aligned}
o(1) & =\tilde{I}_{c}^{\prime}\left(u_{c}\right) u_{c} \\
& =\int_{\mathbb{R}^{3}}\left|\nabla u_{c}\right|^{2}+\left(2 m \mu-\frac{\mu^{2}}{c^{2}}\right) u_{c}^{2}-\left(\frac{q}{c}\right)^{2} u_{c}^{2} \Phi_{u_{c}}^{2}-2 q\left(m-\frac{\mu}{c^{2}}\right) u_{c}^{2} \Phi_{u_{c}}-\left(u_{c}\right)_{+}^{p} d x \\
& =\int_{\mathbb{R}^{3}}\left|\nabla u_{c}\right|^{2}+2 m \mu u_{c}^{2}+2 q m u^{2} \phi_{u}-(u)_{+}^{p} d x+o(1)
\end{aligned}
$$

as $c \rightarrow \infty$. Thus, by (46) and (47), we have $u_{c} \rightarrow u$ in $H^{1}$.

Proposition 15. Let $2<p<3,0<q<\hat{q}_{0}$ and $d \in\left(0, \frac{\hat{d}_{0}}{2}\right)$. For a fixed $c \in\left(\sqrt{\frac{\mu}{m}}, \infty\right)$, suppose that for some $b \in \mathbb{R}$, there exists a sequence $\left\{u_{j}\right\} \subset H_{r}^{1}$ satisfying

$$
\begin{aligned}
& u_{j} \in N_{d}\left(\mathcal{X}_{r}\right), \\
& \left\|\tilde{I}_{c}^{\prime}\left(u_{j}\right)\right\|_{H^{-1}} \rightarrow 0, \\
& \tilde{I}_{c}\left(u_{j}\right) \rightarrow b \text { as } j \rightarrow \infty .
\end{aligned}
$$

Then $b$ is a critical value of $\tilde{I}_{c}$, and the sequence $\left\{u_{j}\right\}_{j=1}^{\infty} \subset H_{r}^{1}$ has a strongly convergent subsequence in $H^{1}$.

Proof. Since $\left\{u_{j}\right\} \subset N_{d}\left(\mathcal{X}_{r}\right)$ is bounded in $H^{1}$, we see that $u_{j}$ converges to $u$ in $L^{s}$ and weakly in $H^{1}$ as $c \rightarrow \infty$, up to a subsequence, where $s \in(2,6)$. It is standard to show that $u$ is a critical point of $\tilde{I}_{c}$. 
We claim that $u_{j} \rightarrow u$ in $H^{1}$. Indeed, by Lemma20 and the fact that $\left\|\tilde{I}_{c}^{\prime}\left(u_{j}\right)\right\|_{H^{-1}} \rightarrow 0$ as $j \rightarrow \infty$, we have

$$
\begin{aligned}
o(1) & =\tilde{I}_{c}^{\prime}\left(u_{j}\right) u_{j} \\
& =\int_{\mathbb{R}^{3}}\left|\nabla u_{j}\right|^{2}+\left(2 m \mu-\frac{\mu^{2}}{c^{2}}\right) u_{j}^{2}-\left(\frac{q}{c}\right)^{2} u_{j}^{2} \Phi_{u_{j}}^{2}-2 q\left(m-\frac{\mu}{c^{2}}\right) u_{j}^{2} \Phi_{u_{j}}-\left|u_{j}\right|^{p} d x \\
& =\int_{\mathbb{R}^{3}}\left|\nabla u_{j}\right|^{2}+\left(2 m \mu-\frac{\mu^{2}}{c^{2}}\right) u_{j}^{2}-\left(\frac{q}{c}\right)^{2} u^{2} \Phi_{u}^{2}-2 q\left(m-\frac{\mu}{c^{2}}\right) u^{2} \Phi_{u}-|u|^{p} d x+o(1)
\end{aligned}
$$

as $j \rightarrow \infty$ and

$$
0=\tilde{I}_{c}^{\prime}(u) u=\int_{\mathbb{R}^{3}}|\nabla u|^{2}+\left(2 m \mu-\frac{\mu^{2}}{c^{2}}\right) u^{2}-\left(\frac{q}{c}\right)^{2} u^{2} \Phi_{u}^{2}-2 q\left(m-\frac{\mu}{c^{2}}\right) u^{2} \Phi_{u}-|u|^{p} d x .
$$

Thus, we deduce that $u_{j} \rightarrow u$ in $H^{1}$ as $j \rightarrow \infty$.

Proposition 16. Let $2<p<3,0<q<\hat{q}_{0}$ and $d \in\left(0, \frac{\hat{d}_{0}}{2}\right)$. Then there exists $\hat{c}_{0}>0$ such that for $c>\hat{c}_{0}, \tilde{I}_{c}$ has a non-trivial critical point $u$ in $N_{d}\left(\mathcal{X}_{r}\right)$ with $\tilde{I}_{c}(u) \leq m_{c}$.

Proof. Assume that $2<p<3,0<q<\hat{q}_{0}$ and $d \in\left(0, \frac{\hat{d}_{0}}{2}\right)$. Suppose $\tilde{I}_{c}^{\prime}(u) \neq 0$ for $u \in N_{d}\left(\mathcal{X}_{r}\right)$ with $\tilde{I}_{c}(u) \leq m_{c}$. By Proposition 1215 we can take a positive constant $\hat{c}_{0}$ such that for $c>\hat{c}_{0}$ and for $0<q<\hat{q}_{0}$,

$$
\begin{gathered}
\alpha_{c} \geq e_{\infty}-\epsilon_{1}, \quad\left|m_{c}-e_{\infty}\right| \leq \epsilon_{1}, \\
\left\|\tilde{I}_{c}^{\prime}(u)\right\|_{H^{-1}} \geq \nu_{0}
\end{gathered}
$$

for $u \in N_{\frac{2}{3} d}\left(\mathcal{X}_{r}\right) \backslash N_{\frac{1}{3} d}\left(\mathcal{X}_{r}\right)$ with $\tilde{I}_{c}(u) \leq m_{c}$, and

$$
\left\|\tilde{I}_{c}^{\prime}(u)\right\|_{H^{-1}} \geq \hat{\sigma}_{c}
$$

for $u \in N_{d}\left(\mathcal{X}_{r}\right)$ with $\tilde{I}_{c}(u) \leq m_{c}$, where $d \in\left(0, \frac{\hat{d}_{0}}{2}\right), \epsilon_{1} \in\left(0, \frac{d \nu_{0}}{6}\right)$, and $\hat{\sigma}_{c}>0$ is a constant depending on $c$. For $u \in N_{d}\left(\mathcal{X}_{r}\right)$ with $\tilde{I}_{c}(u) \leq m_{c}$, we consider the following ODE:

$$
\left\{\begin{array}{l}
\frac{d \eta}{d \tau}=-\varphi_{1}\left(\tilde{I}_{c}(\eta)\right) \varphi_{2}\left(\operatorname{dist}_{H^{1}}\left(\eta, \mathcal{X}_{r}\right)\right) \frac{\tilde{I}_{c}^{\prime}(\eta)}{\left\|\tilde{I}_{c}^{\prime}(\eta)\right\|_{H^{-1}}}, \\
\eta(0, u)=u
\end{array}\right.
$$

where

$$
\operatorname{dist}_{H^{1}}\left(w, \mathcal{X}_{r}\right)=\inf \left\{\|w-v\|_{H^{1}} \mid v \in \mathcal{X}_{r}\right\}
$$

for $w \in H^{1}$, and $\varphi_{1}, \varphi_{2}: \mathbb{R} \rightarrow[0,1]$ are Lipschitz continuous functions such that

$$
\varphi_{1}(\xi)=\left\{\begin{array}{ll}
1 & \text { if } \xi \geq e_{\infty}-\epsilon_{1}, \\
0 & \text { if } \xi \leq e_{\infty}-2 \epsilon_{1},
\end{array} \quad \varphi_{2}(\xi)= \begin{cases}1 & \text { if } \xi \leq \frac{2}{3} d \\
0 & \text { if } \xi \geq d .\end{cases}\right.
$$

Let $T=3 \epsilon_{1} / \hat{\sigma}_{c}$ and $V_{0} \in \mathcal{X}_{r}$. Since $\tilde{I}_{c}\left(\eta\left(\tau, V_{0}\right)\right) \geq \alpha_{c} \geq e_{\infty}-\epsilon_{1}$ for $\tau \in[0, T]$, we deduce that there exists $t_{0} \in[0, T]$ such that

$$
\operatorname{dist}_{H^{1}}\left(\eta\left(t_{0}, V_{0}\right)\right)=\frac{2}{3} d .
$$

Indeed, if $\operatorname{dist}_{H^{1}}\left(\eta\left(\tau, V_{0}\right)\right)<\frac{2}{3} d$ for $\tau \in[0, T]$, by (48) and (50),

$$
\tilde{I}_{c}\left(\eta\left(T, V_{0}\right)\right)=\tilde{I}_{c}\left(V_{0}\right)+\int_{0}^{T} \frac{d}{d \tau} \tilde{I}_{c}\left(\eta\left(\tau, V_{0}\right)\right) d \tau \leq e_{\infty}+\epsilon_{1}-T \hat{\sigma}_{c}=e_{\infty}-2 \epsilon_{1},
$$

which is a contradiction. Assume that $t_{0}$ is the first time that satisfies (51). Since $\left\|\frac{d}{d \tau} \eta\right\|_{H^{1}} \leq 1$, we see that $t_{0} \geq \frac{2}{3} d$ and

$$
\eta\left(\tau, V_{0}\right) \in N_{\frac{2}{3} d}\left(\mathcal{X}_{r}\right) \backslash N_{\frac{1}{3} d}\left(\mathcal{X}_{r}\right) \text { for } \tau \in\left[t_{0}-\frac{1}{3} d, t_{0}\right] .
$$


Then, by (48) and (49), we have

$$
\begin{aligned}
\tilde{I}_{c}\left(\eta\left(T, V_{0}\right)\right) & =\tilde{I}_{c}\left(V_{0}\right)+\int_{0}^{T} \frac{d}{d \tau} \tilde{I}_{c}\left(\eta\left(\tau, V_{0}\right)\right) d \tau \leq e_{\infty}+\epsilon_{1}+\int_{t_{0}-\frac{1}{3} d}^{t_{0}} \frac{d}{d \tau} \tilde{I}_{c}\left(\eta\left(\tau, V_{0}\right)\right) d \tau \\
& =e_{\infty}+\epsilon_{1}-\frac{1}{3} d \nu_{0}<e_{\infty}-\epsilon_{1},
\end{aligned}
$$

which is a contradiction.

Proof of Theorem 2, Let $2<p<3$. By Proposition[16] and the proof of Proposition 14, we prove the existence of a radially symmetric positive solution $v_{c, q}$ of (5) satisfying

$$
\limsup _{c \rightarrow \infty} \tilde{I}_{c}\left(v_{c, q}\right) \leq \inf _{u \in H_{r}^{1}} \tilde{I}_{\infty}(u) \text {. }
$$

By repeating the same procedure in the proof of Proposition 14, we can prove Theorem 2 (ii).

On the other hand, it is known that the ground state solution $w_{0}$ of the equation

$$
-\Delta u+2 m \mu-|u|^{p-2} u=0 \text { in } \mathbb{R}^{3}
$$

is positive, radially symmetric, up to a translation. It is also non-degenerate in the radial class, i.e., $\operatorname{Ker} L_{0}=\{0\}$, where $L_{0}: H_{r}^{1} \rightarrow H_{r}^{-1}$ is the linearized operator of (52) at $w_{0}$, given by $L_{0}(w) \equiv-\Delta w+2 m \mu w-(p-1)\left|u_{0}\right|^{p-2} w$.

Exploiting the non-degeneracy of $w_{0}$, we see from the implicit function theorem that there exists of a family of radially symmetric solutions $w_{\infty, q}$ of (10) for small $q>0$ such that $w_{\infty, q} \rightarrow w_{0}$ as $q \rightarrow 0$ in $H^{1}$. (We refer to [19] for detail.) As a consequence, one can easily see that $w_{\infty, q}$ is also non-degenerate in the radial class for any small fixed $q>0$. Then one can once more invoke the implicit function theorem to find a family of nontrivial radial solutions $w_{c, q}$ of (5) for large value $c>0$ and small $q>0$, which converges in $H^{1}$ to $w_{\infty, q}$ as $c \rightarrow \infty$. This proves Theorem 2 (i).

\section{Appendix A. Basic estimates}

Here, we provide with several basic estimates, which are repeatedly invoked in the proofs of main theorems.

Lemma 17. Let $u \in H^{1}$. Then we have

$$
\left\|\phi_{u}\right\|_{D^{1,2}} \leq C q m\|u\|_{H^{1}}^{2}
$$

where $C$ is a positive constant.

Proof. Let $u \in H^{1}$. Since $\phi_{u}$ satisfies

$$
-\Delta \phi_{u}=-q m u^{2} \text { in } \mathbb{R}^{3},
$$

we have

$$
\int_{\mathbb{R}^{3}}\left|\nabla \phi_{u}\right|^{2} d x=-q m \int_{\mathbb{R}^{3}} u^{2} \phi_{u} d x \leq q m\left\|\phi_{u}\right\|_{L^{6}}\left\|u^{2}\right\|_{L^{6 / 5}} \leq C q m\left\|\phi_{u}\right\|_{D^{1,2}}\|u\|_{H^{1}}^{2},
$$

where $C$ is a positive constant. This implies the result.

Lemma 18. Let $u \in H^{1}$. For $c>\sqrt{\frac{\mu}{m}}$, we have

$$
\left\|\Phi_{u}\right\|_{D^{1,2}} \leq C q\left(m-\frac{\mu}{c^{2}}\right)\|u\|_{H^{1}}^{2}
$$

where $C$ is a positive constant.

Proof. Let $u \in H^{1}$. Since $\Phi_{u}$ satisfies

$$
-\Delta \Phi_{u}+\left(\frac{q}{c}\right)^{2} u^{2} \Phi_{u}=-q\left(m-\frac{\mu}{c^{2}}\right) u^{2} \text { in } \mathbb{R}^{3},
$$

and

$$
\left\|u^{2} \Phi_{u}\right\|_{L^{1}} \leq\left\|\Phi_{u}\right\|_{L^{6}}\left\|u^{2}\right\|_{L^{6 / 5}}=\left\|\Phi_{u}\right\|_{L^{6}}\|u\|_{L^{12 / 5}}^{2} \leq C\left\|\Phi_{u}\right\|_{D^{1,2}}\|u\|_{H^{1}}^{2},
$$


we have for $c>\sqrt{\frac{\mu}{m}}$,

$$
\begin{aligned}
\left\|\Phi_{u}\right\|_{D^{1,2}}^{2}=\int_{\mathbb{R}^{3}}\left|\nabla \Phi_{u}\right|^{2} d x & \leq-q\left(m-\frac{\mu}{c^{2}}\right) \int_{\mathbb{R}^{3}} u^{2} \Phi_{u} \\
& \leq C q\left(m-\frac{\mu}{c^{2}}\right)\|u\|_{H^{1}}^{2}\left\|\Phi_{u}\right\|_{D^{1,2}},
\end{aligned}
$$

where $C$ is a positive constant. This implies the result.

Lemma 19. Let $v, w \in H^{1}$. Then we have

$$
\left\|\phi_{v}-\phi_{w}\right\|_{D^{1,2}} \leq C\|v+w\|_{H^{1}}\|v-w\|_{H^{1}}
$$

where $C=C(q, m)$ is a positive constant.

Proof. We note that for $v, w \in H^{1}$,

$$
-\Delta\left(\phi_{v}-\phi_{w}\right)=-q m(v-w)(v+w) \text { in } \mathbb{R}^{3} .
$$

Then we have

$$
\left\|\phi_{v}-\phi_{w}\right\|_{D^{1,2}} \leq C\|v+w\|_{H^{1}}\|v-w\|_{H^{1}}
$$

where $C=C(q, m)$ is a positive constant.

Lemma 20. Let $v, w \in H^{1}$. Then for $c>\sqrt{\frac{\mu}{m}}$, we have

$$
\left\|\Phi_{v}-\Phi_{w}\right\|_{D^{1,2}} \leq C\left(\|v\|_{H^{1}}^{2}+1\right)\|v+w\|_{H^{1}}\|v-w\|_{L^{3}}
$$

where $C=C(q, m, \mu)$ is a positive constant.

Proof. Since $\Phi_{u}$ satisfies

$$
-\Delta \Phi_{u}+\frac{q^{2}}{c^{2}} u^{2} \Phi_{u}=-q\left(m-\frac{\mu}{c^{2}}\right) u^{2} \text { in } \mathbb{R}^{3},
$$

we have

$$
-\Delta\left(\Phi_{v}-\Phi_{w}\right)+\frac{q^{2}}{c^{2}} w^{2}\left(\Phi_{v}-\Phi_{w}\right)=-\frac{q^{2}}{c^{2}}\left(v^{2}-w^{2}\right) \Phi_{v}-q\left(m-\frac{\mu}{c^{2}}\right)\left(v^{2}-w^{2}\right) \text { in } \mathbb{R}^{3} .
$$

Multiplying $\left(\Phi_{v}-\Phi_{w}\right)$ to the above equation and then integrating over $\mathbb{R}^{3}$, we have

$$
\begin{aligned}
\int_{\mathbb{R}^{3}}\left|\nabla\left(\Phi_{v}-\Phi_{w}\right)\right|^{2} d x \\
\leq \int_{\mathbb{R}^{3}}-\frac{q^{2}}{c^{2}}\left(v^{2}-w^{2}\right) \Phi_{v}\left(\Phi_{v}-\Phi_{w}\right)-q\left(m-\frac{\mu}{c^{2}}\right)\left(v^{2}-w^{2}\right)\left(\Phi_{v}-\Phi_{w}\right) d x \\
\leq \frac{q^{2}}{c^{2}}\|v+w\|_{L^{3}}\|v-w\|_{L^{3}}\left\|\Phi_{v}\right\|_{L^{6}}\left\|\Phi_{v}-\Phi_{w}\right\|_{L^{6}} \\
\quad \quad+q\left(m-\frac{\mu}{c^{2}}\right)\|v+w\|_{L^{2}}\|v-w\|_{L^{3}}\left\|\Phi_{v}-\Phi_{w}\right\|_{L^{6}} \\
\leq C_{1}\left(\left\|\Phi_{v}\right\|_{D^{1,2}}+1\right)\left\|\Phi_{v}-\Phi_{w}\right\|_{D^{1,2}}\|v+w\|_{H^{1}}\|v-w\|_{L^{3}} .
\end{aligned}
$$

where $C_{1}=C_{1}(q, m, \mu)$ is a positive constant. Then, by Lemma 18, for $c>\sqrt{\frac{\mu}{m}}$,

$$
\left\|\Phi_{v}-\Phi_{w}\right\|_{D^{1,2}} \leq C\left(\|v\|_{H^{1}}^{2}+1\right)\|v+w\|_{H^{1}}\|v-w\|_{L^{3}}
$$

where $C=C(q, m, \mu)$ is a positive constant.

Lemma 21.

$$
\left\|\Phi_{v}-\phi_{w}\right\|_{D^{1,2}} \leq C\left(\frac{1}{c^{2}}\left(\|v\|_{H^{1}}^{2}+1\right)\|v\|_{H^{1}}^{2}+\|v+w\|_{H^{1}}\|v-w\|_{L^{3}}\right),
$$

where $C=C(q, m, \mu)$ is a positive constant. 
Proof. Since $\phi_{w}$ and $\Phi_{v}$ satisfy

$$
-\Delta \phi_{w}=-q m w^{2} \text { in } \mathbb{R}^{3} \text { and }-\Delta \Phi_{v}=-\frac{q^{2}}{c^{2}} v^{2} \Phi_{v}-q\left(m-\frac{\mu}{c^{2}}\right) v^{2} \text { in } \mathbb{R}^{3}
$$

respectively, we have

$$
-\Delta\left(\Phi_{v}-\phi_{w}\right)=-\frac{q^{2}}{c^{2}} v^{2} \Phi_{v}+q \frac{\mu}{c^{2}} v^{2}-q m\left(v^{2}-w^{2}\right) \text { in } \mathbb{R}^{3} .
$$

We multiply $\left(\Phi_{v}-\phi_{w}\right)$ to the above equation and integrate over $\mathbb{R}^{3}$ to deduce

$$
\begin{aligned}
& \int_{\mathbb{R}^{3}}\left|\nabla\left(\Phi_{v}-\phi_{w}\right)\right|^{2} d x \\
& =\frac{1}{c^{2}} \int_{\mathbb{R}^{3}}\left(-q^{2} v^{2} \Phi_{v}+q \mu v^{2}\right)\left(\Phi_{v}-\phi_{w}\right) d x-q m \int_{\mathbb{R}^{3}}\left(v^{2}-w^{2}\right)\left(\Phi_{v}-\phi_{w}\right) d x \\
& \leq \frac{1}{c^{2}}\left\|\Phi_{v}-\phi_{w}\right\|_{L^{6}}\left(q^{2}\left\|\Phi_{v}\right\|_{L^{6}}\left\|v^{2}\right\|_{L^{3 / 2}}+q \mu\left\|v^{2}\right\|_{L^{6 / 5}}\right)+q m\left\|\Phi_{v}-\phi_{w}\right\|_{L^{6}}\|v+w\|_{L^{2}}\|v-w\|_{L^{3}} \\
& \leq C_{1}\left\|\Phi_{v}-\phi_{w}\right\|_{D^{1,2}}\left(\frac{1}{c^{2}}\left(\left\|\Phi_{v}\right\|_{D^{1,2}}\|v\|_{H^{1}}^{2}+\|v\|_{H^{1}}^{2}\right)+\|v+w\|_{H^{1}}\|v-w\|_{L^{3}}\right),
\end{aligned}
$$

where $C_{1}=C_{1}(q, m, \mu)$ is a positive constant. Then, by Lemma 18, we have

$$
\left\|\Phi_{v}-\phi_{w}\right\|_{D^{1,2}} \leq C\left(\frac{1}{c^{2}}\left(\|v\|_{H^{1}}^{2}+1\right)\|v\|_{H^{1}}^{2}+\|v+w\|_{H^{1}}\|v-w\|_{L^{3}}\right),
$$

where $C=C(q, m, \mu)$ is a positive constant.

\section{REFERENCES}

[1] A. Azzollini, A. Pomponio, Ground state solutions for the nonlinear Schrödinger-Maxwell equations. J. Math. Anal. Appl. 345 (2008), no. 1, 90-108.

[2] A. Azzollini, A. Pomponio, Ground state solutions for the nonlinear Klein-Gordon-Maxwell equations. Topol. Methods Nonlinear Anal. 35 (2010), no. 1, 33-42.

[3] A. Azzollini, L. Pisani, A. Pomponio, Improved estimates and a limit case for the electrostatic Klein-GordonMaxwell system. Proc. Roy. Soc. Edinburgh Sect. A 141 (2011), no. 3, 449-463.

[4] P. Bechouche, N. J. Mauser, S. Selberg, Nonrelativistic limit of Klein-Gordon-Maxwell to Schrödinger-Poisson. Amer. J. Math. 126 (2004), no. 1, 31-64.

[5] J. Byeon, L. Jeanjean, Standing waves for nonlinear Schrödinger equations with a general nonlinearity. Arch. Ration. Mech. Anal. 185 (2007), no. 2, 185-200.

[6] V. Benci, D. Fortunato, Solitary waves of the nonlinear Klein-Gordon equation coupled with the Maxwell equations. Rev. Math. Phys. 14 (2002), no. 4, 409-420.

[7] G. M. Coclite, A multiplicity result for the nonlinear Schrödinger-Maxwell equations. Commun. Appl. Anal. 7 (2003), no. 2-3, 417-423.

[8] M. Colin, T. Watanabe, Standing waves for the nonlinear Schrödinger equation coupled with the Maxwell equation. Nonlinearity 30 (2017), no. 5, 1920-1947.

[9] W. Choi, Y. Hong, J. Seok, Optimal convergence rate and regularity of nonrelativistic limit for the nonlinear pseudo-relativistic equations. J. Funct. Anal. 274 (2018), no. 3, 695-722.

[10] W. Choi, J. Seok, Nonrelativistic limit of standing waves for pseudo-relativistic nonlinear Schrödinger equations. J. Math. Phys. 57 (2016), no. 2, 021510, 15 pp.

[11] T. D'Aprile, D. Mugnai, Solitary waves for nonlinear Klein-Gordon-Maxwell and Schrödinger-Maxwell equations. Proc. Roy. Soc. Edinburgh Sect. A 134 (2004), no. 5, 893-906.

[12] T. D'Aprile, D. Mugnai, Non-existence results for the coupled Klein-Gordon-Maxwell equations. Adv. Nonlinear Stud. 4 (2004), no. 3, 307-322.

[13] P.-L. Lions, The concentration-compactness principle in the calculus of variations. The locally compact case. II. Ann. Inst. H. Poincaré Anal. Non Linéaire 1 (1984), no. 4, 223-283.

[14] S. Machihara, K. Nakanishi, T. Ozawa, Nonrelativistic limit in the energy space for nonlinear Klein-Gordon equations. Math. Ann. 322 (2002), no. 3, 603-621.

[15] N. Masmoudi, K. Nakanish, From nonlinear Klein-Gordon equation to a system of coupled nonlinear Schrödinger equations. Math. Ann. 324 (2002), no. 2, 359-389.

[16] N. Masmoudi, K. Nakanishi, Nonrelativistic limit from Maxwell-Klein-Gordon and Maxwell-Dirac to PoissonSchrödinger. Int. Math. Res. Not. (2003), no. 13, 697-734.

[17] K. Nakanishi, Nonrelativistic limit of scattering theory for nonlinear Klein-Gordon equations. J. Differential Equations 180 (2002), no. 2, 453-470.

[18] R. S. Palais, The principle of symmetric criticality. Comm. Math. Phys. 69 (1979), no. 1, 19-30. 
[19] D. Ruiz, The Schrödinger-Poisson equation under the effect of a nonlinear local term. J. Funct. Anal. 237 (2006), no. $2,655-674$.

[20] Q. Han, F. Lin, Elliptic Partial Differential Equations, second edition, Courant Lecture Notes in Mathematics, vol. 1, Courant Institute of Mathematical Sciences, American Mathematical Society, New York, Providence, RI, 2011.

[21] F. Wang, Ground-state solutions for the electrostatic nonlinear Klein-Gordon-Maxwell system. Nonlinear Anal. 74 (2011) 4796-4803.

Department of Mathematical Sciences, Kaist, Daejeon 34141, Republic of Korea Email address: sdjin@kaist.ac.kr

Department of Mathematics, Kyonggi University, Suwon 16227, Republic of Korea Email address: jmseok@kgu.ac.kr 